\title{
Laminar Flame Propagation in a Premixed Particle Cloud: Effect of Vaporization Rate
}

\author{
C. Vázquez-Espí
}

Department of Applied Mathematics, E. T. S. de Ingeniería Aeronáutica y del Espacio, Universidad Politécnica de Madrid (UPM), Madrid, Spain

\begin{abstract}
The propagation of a flame in a mixture of air and small solid fuel particles is analyzed. It is assumed that solid particles suffer a vaporization, after which the resulting gaseous fuel mixes with air and a premixed combustion occurs. The combustion reaction is modeled by a global reaction of Arrhenius type with large activation energy and the analysis is carried out using large activation energy asymptotics. The structure of the flow is similar to a classical premixed flame, but with some particular features due to the vaporization, whose characteristic time, through the appropriate Damköhler number, plays an important role. Lean and rich mixtures, defined from the solid fuel content, are considered, as well as the effect of Lewis numbers and heat of vaporization. The analysis shows that rich mixtures can be lean or rich in the gaseous phase depending on the value of the vaporization Damköhler number. In all regimes the structure of flow and the flame speed are obtained.
\end{abstract}

\section{ARTICLE HISTORY}

Received 30 October 2018

Revised 11 March 2019

Accepted 11 March 2019

\section{KEYWORDS}

Vaporization; Particle cloud; Flame propagation; Flame speed; Volatile fuel

\section{Introduction}

The analysis of flame propagation in a cloud of solid fuel particles has received a lot of attention for a long time in order to improve the knowledge of dust explosions and fires in industrial facilities as coal plants, cereal silos or mills (Eckhoff, 1997). More recently, the need for new energy resources has increased the interest in the use of biomass particles in small power plants. In several works Bidabadi et al. (2015, and the references therein) have considered the lycopodium dust flame with the double aim of studying the mechanisms of explosions and flame propagation in dust clouds, and the application of microscale biomass to small power plants as Stirling engines.

The aim of this work is to study the flame propagation in a premixed mixture of oxidant and gaseous fuel resulting from the vaporization of micro organic particles, as starch dust or lycopodium whose diameters are around $30 \mu \mathrm{m}$ (Proust, 2006b), focusing on the role of the characteristic time and heat of vaporization. A question that arises is to what extent the premixed flame model is applicable. An isolated particle typically burns with an envelope diffusion flame surrounding it. In a cloud of particles, the gaseous fuel from the condensed phase can mix with the air before burning if the corresponding diffusion flame radius is larger than the characteristic distance between particles, which 
requires a large enough density of particles. In the analysis of spray combustion, Williams (1985, chap. 11) has shown that cloud-burning mode occurs if the equivalence ratio of the initial mixture is greater than, approximately, 0.7 , and that homogeneous combustion occurs in a monodisperse spray if the initial droplet diameter is about $20 \mu \mathrm{m}$. Nakamura et al. (2005) have performed DNS to study the spray combustion mechanism showing that diffusion-like combustion occurs for large droplets, diameter $\sim 100 \mu \mathrm{m}$, while for small droplets, diameter $\sim 30 \mu \mathrm{m}$, premixed-like combustion appears. A similar claim has been done by Gao et al. (2015). Many studies on flame propagation in small particle clouds have acknowledged the former results and considered that combustion occurs in premixed mode (Bidabadi et al., 2010; Rockwell and Rangwala, 2013; Seshadri et al., 1992).

For small particles of coal, Bermúdez et al. $(2007,2011)$ have established that gas-phase reactions do not occur in diffusion flames around the particles. Therefore the gaseous fuel produced by the vaporization is not burned around the particles but mixed with the air. Since the initial mixture of solid particles is assumed to be homogeneous, the resulting fuel air mixture is also homogeneous, through which a premixed flame can propagate. Proust (2006a, 2006b) has experimentally observed laminar flame propagation in several dust air mixtures, with mean particle diameter between $28 \mu \mathrm{m}$ and $42 \mu \mathrm{m}$ and low vaporization temperature, supporting the idea of a great similarity with premixed gaseous flames.

Two reactions occur simultaneously in the mixture: vaporization and combustion. The first one will be modeled by a reaction rate depending on temperature through a Heaviside function (Bidabadi et al., 2015, 2010), although other approaches are possible (Seshadri et al., 1992), while the combustion will be modeled by a global reaction of Arrhenius type, with activation energy much larger than the thermal energy. This will allow us to use asymptotic techniques in the analysis. Each reaction has its own characteristic time and Damköhler number, defined as the ratio of the residence time to the reaction time. Thus we will define two Damköhler numbers, $D_{v}$ and $D_{a}$ associated, respectively, to the vaporization and combustion reactions. $D_{v}$ determines the rate at which the solid particles are converted into gaseous fuel. For very large values of $D_{v}$ the vaporization is very fast and we find the classical premixed flame. However for order unity, or smaller, values of $D_{v}$ the vaporization is not completed upstream of the reaction zone, but it continues in the convective zone located downstream. In previous works (Bidabadi et al., 2015, 2010; Seshadri et al., 1992) the analysis of this zone appears to be incomplete. The value of $D_{v}$, which determines the distribution of gaseous fuel, has an important effect over the flow structure and flame speed, hence we will analyze the problem in the range of $D_{v}$ of order unity and determine the structure of the flow and the flame speed. Our analysis will include the effects of heat of vaporization and Lewis number, often neglected in other works.

This problem could be addressed from very detailed models available in the literature (e.g., Cloney et al., 2018; Krazinski et al., 1979; Slezak et al., 1985; Smoot and Horton, 1977), but, due to their high degree of complexity, it is necessary to treat them by a nontrivial numerical approach. Instead, we will consider a simplified model in order to use as many analytical tools as possible, although the full solution will also require numerics but to a lower level. This approach will allow us to give a clear picture of the role of $D_{v}$ that otherwise could be hidden. 


\section{Governing equations}

Let us consider a uniform mixture of air and solid fuel particles. The Stokes number of these particles, defined as the ratio between the particle relaxation time and the residence time in a typical combustion chamber, is small, around $10^{-2}$, therefore we can assume they behave as non-inertial particles and move with the same velocity as the gas. The temperature inside the small particles considered in this work is assumed to be uniform and equal to the local gas temperature (Proust, 2006a). When the temperature is larger than a threshold temperature, or vaporization temperature, we assume that all solid material in each particle is converted by vaporization into gaseous fuel, although not instantaneously, which mixes perfectly with the air resulting in a homogeneous reactive mixture.

The species in the mixture are fuel in solid and gas phases, $\mathrm{F}(\mathrm{s})$ and $\mathrm{F}(\mathrm{g})$, oxygen, $\mathrm{O}(\mathrm{g})$, nitrogen, $\mathrm{N}(\mathrm{g})$, and the products of combustion collectively represented by $\mathrm{P}(\mathrm{g})$. The mass fractions are $Y_{s}, Y_{g}, Y_{o}, Y_{n}$ and $Y_{p}$, respectively.

The vaporization is modeled by the reaction $\mathrm{F}(\mathrm{s}) \rightarrow \mathrm{F}(\mathrm{g})-M_{g} Q_{v}$, where $Q_{v}$ is the heat required to vaporize the unit mass of solid fuel and $M_{g}$ is the molecular mass of the fuel. The mass rate of this reaction is assumed to be given by

$$
W_{v}=-M_{g} \frac{\rho Y_{s}}{M_{g}} \frac{1}{t_{v}} H\left(T-T_{v}\right),
$$

where $\rho$ is the mixture density, $H$ is the Heaviside function, $T_{v}$ is the threshold temperature for vaporization and $t_{v}$ is the vaporization characteristic time.

The chemical reaction between gaseous fuel and oxygen is modeled by a single irreversible reaction $v_{g} \mathrm{~F}(\mathrm{~g})+v_{o} \mathrm{O}(\mathrm{g}) \rightarrow v_{p} \mathrm{P}(\mathrm{g})+v_{g} M_{g} \mathrm{Q}$, where $v_{g}, v_{o}$ and $v_{p}$ are the stoichiometric coefficients and $Q$ stands for the heat released by unit mass of fuel. The gaseous fuel consumption rate is given by an Arrhenius law, with activation energy $E$ much larger than the thermal energy $R T$, written in the form:

$$
W_{g}=-v_{g} M_{g} \frac{\rho Y_{g}}{M_{g}} \frac{\rho Y_{o}}{M_{o}} B_{1} \mathrm{e}^{-E / R T},
$$

where $B_{1}$ is the pre-exponential factor that we will assume to be independent of temperature.

For simplicity we assume that the air composition by volume is $21 \%$ oxygen and $79 \%$ nitrogen, with a average molecular mass equal to $28.84 \times 10^{-3} \mathrm{~kg} / \mathrm{mol}$ and a relation between the mass fraction of nitrogen and oxygen equal to 3.292 . The equivalence ratio, $\phi$, and mass fractions of the reactive species in the fresh mixture, in terms of $\phi$, are given by

$$
\phi=v \frac{Y_{s, u}}{Y_{o, u}}, \quad Y_{s, u}=\frac{\phi}{\phi+4.292 v}, \quad Y_{o, u}=\frac{v}{\phi+4.292 v}, \quad Y_{g, u}=0
$$

where $v=v_{o} M_{o} / v_{g} M_{g}$ is the mass of oxygen required to burn the unit mass of fuel. This classical definition of the equivalence ratio involves the solid fuel. However, the chemical reaction takes place in the gaseous phase and the amount of gaseous fuel depends on the 
vaporization rate. If this process is very slow a rich mixture can become lean when we account for the amount of gaseous fuel available to be burned at the flame. Thus, in Section 3.3, when analyzing rich mixtures, we will distinguish between gas-phase rich or lean mixtures.

In order to formulate the problem in a suitable way for an analytic treatment we will made the following assumptions:

(1) The flow is one-dimensional and steady. The pressure is constant.

(2) The solid particles have the same temperature and velocity as those of the gas phase.

(3) The gas phase behaves as a mixture of ideal gases with a constant mean molecular mass.

(4) The mass diffusion and heat conduction fluxes can be described by Fick's and Fourier's laws, respectively. Radiation heat transfer is neglected.

With these assumptions the equations describing the vaporization of solid fuel and the combustion of the gaseous mixture are as follows (Rockwell and Rangwala, 2013; Seshadri et al., 1992):

$$
\begin{gathered}
m \frac{\mathrm{d} Y_{s}}{\mathrm{~d} x}=W_{v} \\
m \frac{\mathrm{d} Y_{g}}{\mathrm{~d} x}=\frac{\mathrm{d}}{\mathrm{d} x}\left(\rho D_{g} \frac{\mathrm{d} Y_{g}}{\mathrm{~d} x}\right)-W_{v}+W_{g} \\
m \frac{\mathrm{d} Y_{o}}{\mathrm{~d} x}=\frac{\mathrm{d}}{\mathrm{d} x}\left(\rho D_{0} \frac{\mathrm{d} Y_{o}}{\mathrm{~d} x}\right)+v W_{g} \\
m c_{p} \frac{\mathrm{d} T}{\mathrm{~d} x}=\frac{\mathrm{d}}{\mathrm{d} x}\left(\lambda \frac{\mathrm{d} T}{\mathrm{~d} x}\right)+Q_{v} W_{v}-Q W_{g} \\
\rho T=\rho_{u} T_{i} .
\end{gathered}
$$

In this model the mass flux $m$ is assumed to be constant and given by $m=\rho_{u} U_{p}$, where $U_{p}$ stands for the burning velocity, or flame speed. The diffusion coefficients of gaseous fuel and oxygen, $D_{g}$ and $D_{o}$, respectively, and the thermal conductivity, $\lambda$, are functions of temperature to be defined later and the specific heat, $c_{p}$, is considered constant and equal for all species.

These equations must be supplemented with the following boundary conditions:

$$
\begin{gathered}
x \rightarrow-\infty: \quad Y_{s}=Y_{s, t}, Y_{g}=0, Y_{o}=Y_{o, t}, T=T_{t} ; \\
x \rightarrow+\infty: \quad \frac{\mathrm{d} Y_{s}}{\mathrm{~d} x}=\frac{\mathrm{d} Y_{g}}{\mathrm{~d} x}=\frac{\mathrm{d} Y_{o}}{\mathrm{~d} x}=\frac{\mathrm{d} T}{\mathrm{~d} x}=0 .
\end{gathered}
$$


Moreover, due to the irreversible reaction between oxygen and fuel, the burned gas must be in chemical equilibrium, at least in first approximation, which requires that at least one reactant must be completely consumed and, hence, in first approximation $Y_{g} Y_{o} \rightarrow 0$ as $x \rightarrow+\infty$.

\section{Conservation laws}

From suitable linear combinations of Equations (2.4)-(2.7) we can obtain equations free from reaction terms, that, after integrating between $\pm \infty$ and taking into account the boundary conditions, lead to the following conservation laws:

$$
\begin{gathered}
Y_{o, u}-Y_{o, b}=v\left(Y_{s, u}-Y_{s, b}-Y_{g, b}\right), \\
Q\left(Y_{s, u}-Y_{s, b}-Y_{g, b}\right)=c_{p}\left(T_{b}-T_{u}\right)+Q_{v}\left(Y_{s, u}-Y_{s, b}\right),
\end{gathered}
$$

where the subscript $b$ makes reference to the conditions at the burned gas side, $x \rightarrow+\infty$. Equation (2.11) represents the stoichiometric relation between the consumption of both oxidant and fuel, while (2.12) represents the energy balance between the thermal enthalpy of the mixture, the heat released by the combustion and the energy required for the vaporization. Since this process occurs only if the temperature is greater or equal to $T_{v}$, we need to burn a certain amount of gaseous fuel such that, at some point upstream of the flame, the temperature reaches the value $T_{v}$ and the vaporization can be sustained. If the temperature is lower than $T_{v}$ everywhere the fresh mixture will remain unchanged.

When the final temperature $T_{b}$ is larger than $T_{v}$ all solid fuel is converted into gaseous fuel, hence $Y_{s, b}=0$. If the mixture is lean, $\phi<1$, all gaseous fuel is burned and $Y_{g, b}=0$, while when the mixture is rich, $\phi>1$, the oxygen is completely depleted and $Y_{o, b}=0$. Therefore we can identify the following regimes:

\section{Rich mixture $\left(1<\phi<\phi_{1}\right)$ and full vaporization $\left(T_{b}>T_{v}\right)$}

The corresponding burned side values are given by

$$
Y_{o, b}=Y_{s, b}=0, \quad Y_{g, b}=Y_{s, u}-\frac{Y_{o, u}}{v}=\frac{\phi-1}{\phi+4.292 v}, T_{b}=T_{u}+\frac{1}{c_{p}} \frac{Q-Q_{v} \phi}{\phi+4.292 v} .
$$

Note that $Y_{g, b}$ is increasing with $\phi$ while $T_{b}$ is decreasing. Hence this regime only exists for $1<\phi<\phi_{1}$, where $\phi_{1}$ is the value at which $T_{b}=T_{v}$.

If $\phi>\phi_{1}$ the final temperature determined by (2.13) results lower than $T_{v}$ and, hence, the solid fuel is not completely consumed, $Y_{s, b} \neq 0$, giving rise to a new regime characterized by an incomplete vaporization and $T_{b}=T_{v}$. For sufficiently large values of $\phi$ the amount of oxygen in the fresh side is not enough to burn the minimum amount of fuel required to rise the temperature from $T_{u}$ to $T_{v}$. The maximum value of $\phi, \phi_{2}$, for which is possible to reach $T_{v}$ is determined by the conditions $T_{b}=T_{v}, Y_{o, b}=0$ and $Y_{g, b}\left(\phi_{2}\right)=0$. Therefore, if $\phi>\phi_{2}$ the vaporization cannot be sustained in a steady flow. 


\section{Lean mixture $\left(\phi_{0}<\phi<1\right)$ and full vaporization $\left(T_{b}>T_{v}\right)$}

In this regime the burned side values are given by:

$$
Y_{s, b}=Y_{g, b}=0, \quad Y_{o, b}=Y_{o, u}-v Y_{s, u}=\frac{v(1-\phi)}{\phi+4.292 v}, T_{b}=T_{u}+\frac{1}{c_{p}} \frac{\left(Q-Q_{v}\right) \phi}{\phi+4.292 v} .
$$

Now $Y_{o, b}$ is decreasing with $\phi$ while $T_{b}$ is increasing. This regime only exists in the range $\phi_{0}<\phi<1$, where $\phi_{0}$ is defined by the condition $T_{b}\left(\phi_{0}\right)=T_{v}$.

If $\phi<\phi_{0}$ the amount of solid fuel is smaller than the minimum required to reach $T_{v}$.

Hence, the conservation laws show that a steady flame propagation only occurs in the range of equivalence ratios $\phi_{0}<\phi<\phi_{2}$. Figure 1 shows mass fractions $Y_{s, u}, Y_{s, u}, Y_{o, u}, Y_{o, b}$ and $Y_{g, b}$ and the burned side temperature $T_{b}$ (in the nondimensional form $10 c_{p}\left(T_{b}-T_{v}\right) / Q$ ) as functions of the equivalence ratio $\phi$. For similar values of the parameters $v, Q / Q_{v}$ and $c_{p}\left(T_{v}-T_{u}\right) / Q$ to those considered in previous works, the values of $\phi$ determining the three regimes are $\phi_{0} \sim 0.5, \phi_{1} \sim 7.3$ and $\phi_{2} \sim 19.4$. Therefore the incomplete vaporization regime occurs for very large values of the equivalence ratio and, hence, has a marginal character. For this reason it will not be considered in the rest of this work, and we will restrict to values of $\phi$ in the range $\left[\phi_{0}, \phi_{1}\right]$, for which the vaporization is always complete $\left(Y_{s, b}=0\right)$.

The temperature $\mathrm{Tb}$ is maximum for $\Phi \frac{1 / 4}{1}$ (stoichiometric conditions) and decreases when $\Phi$ moves from 1 to $\Phi 0$ or $\Phi 1$. Note that the final temperature, $T_{b}$, will not be necessarily the flame temperature. For rich mixtures there is an oxygen free region, located downstream the flame, where the temperature is greater than $T_{v}$, so that the vaporization continues and the temperature decreases. Therefore the flame temperature will be larger than $T_{b}$. If the mixture is lean the temperature downstream the flame increases due to the combustion of the gaseous fuel produced by the vaporization of the solid fuel remaining. The flame temperature will then be lower than $T_{b}$.

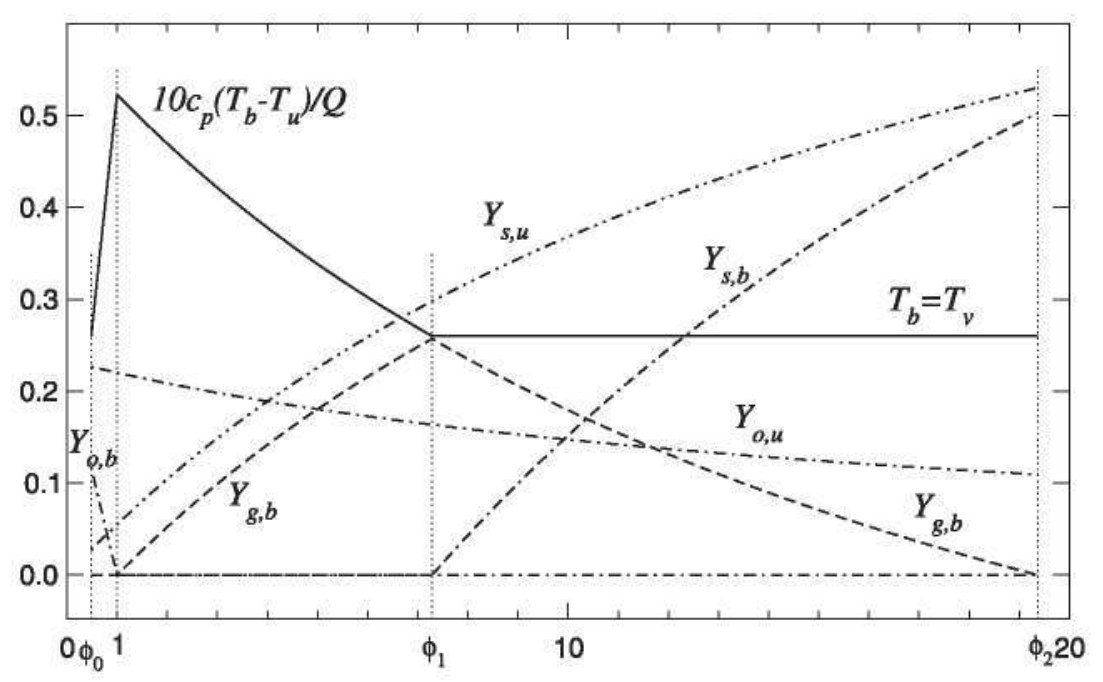

Figure 1. Values of $Y_{s, u}, Y_{s, u}, Y_{o, u}, Y_{o, b}, Y_{g, b}$ and $T_{b}$ as functions of $\phi$. 


\section{Nondimensional equations}

To nondimensionalize the problem it is convenient to introduce the following definitions and parameters: thermal diffusivity, $D_{T}=\lambda / \rho c_{p}$; Lewis number of species $i$, $L_{i}=\left(D_{T, u} / D_{i, u}\right)$; nondimensional vaporization heat, $q=Q_{v} / Q$; preheat zone thickness, $l_{T}=D_{T, u} / U_{p}$; residence time in the preheat zone: $t_{r}=l_{T} / U_{p}=D_{T, u} / U_{p}^{2}$; and adiabatic temperature, $T_{m}=T_{u}+\left(Q Y_{s, u} / c_{p}\right) \min \left(1, \phi^{-1}\right)$. Note that in a lean mixture $T_{m}$ corresponds to the adiabatic temperature obtained when all allowable solid fuel is burned, while in a rich mixture the deficient reactant is the oxygen and then the adiabatic temperature is given by $T_{m}=T_{u}+\left(Q / c_{p}\right)\left(Y_{s, u} / \phi\right)=T_{u}+\left(Q / c_{p}\right)\left(Y_{o, u} / v\right)$.

The nondimensional temperature, mass fractions and space coordinate are defined by:

$$
\theta=\frac{T-T_{u}}{T_{m}-T_{u}}, \quad y_{s}=\frac{Y_{s}}{Y_{s, u}}, \quad y_{g}=\frac{Y_{g}}{Y_{s, u}}, \quad y_{o}=\frac{Y_{o}}{v Y_{s, u}}, \quad \xi=\int_{0}^{x} \frac{\rho}{\rho_{u}} \frac{\mathrm{d} x}{l_{T}} .
$$

With these parameters and variables the reaction terms are written as

$$
\begin{gathered}
W_{v}=-\rho_{u} Y_{s, u} \frac{\rho}{\rho_{u}} y_{s} \frac{1}{t_{v}} H\left(\theta-\theta_{v}\right), \\
W_{g}=-\rho_{u} Y_{s, u} \frac{\rho}{\rho_{u}} B(T) \mathrm{e}^{-T_{a} / T_{f}} y_{g} y_{o} \exp \left[\frac{\beta\left(\theta-\theta_{f}\right)}{1+\gamma\left(\theta-\theta_{f}\right)}\right],
\end{gathered}
$$

where $T_{f}$ is the flame temperature, which, according to the large activation energy limit, is assumed to be much smaller than the activation temperature, i.e., $T_{f} \ll T_{a}=E / R$; the heat released parameter $\gamma=\left(T_{m}-T_{u}\right) / T_{f}$ is of order unity; $\beta=T_{a}\left(T_{m}-T_{u}\right) / T_{f}^{2} \gg 1$ is the Zel'dovich number; and $B(T)=v_{o} M_{g}^{-1} Y_{s, u} \rho_{u} T_{u} T^{-1} B_{1}$ is a modified pre-exponential factor. The chemical time associated to the combustion reaction is defined by $t_{q}^{-1}=B\left(T_{f}\right) \mathrm{e}^{-T_{a} / T_{f}}$.

In order to simplify the mathematical problem we will assume that $D_{i} \sim T^{2}$ and $\lambda \sim T$. Taking into account that $\rho \sim 1 / T$ we find that $\rho^{2} D_{i}=\rho_{u}^{2} D_{i, u}$ and $\rho \lambda=\rho_{u} \lambda_{u}$ and, as consequence, the ratio of thermal and mass diffusivities is independent of the temperature and equal to the Lewis number. The Equations (2.4)-(2.7) written in nondimensional form read:

$$
\begin{aligned}
& \frac{\mathrm{d} y_{s}}{\mathrm{~d} \xi}=w_{v} \\
& \frac{\mathrm{d} y_{g}}{\mathrm{~d} \xi}=\frac{1}{L_{g}} \frac{\mathrm{d}^{2} y_{g}}{\mathrm{~d} \xi^{2}}-w_{v}+w_{g}, \\
& \frac{\mathrm{d} y_{o}}{\mathrm{~d} \xi}=\frac{1}{L_{o}} \frac{\mathrm{d}^{2} y_{o}}{\mathrm{~d} \xi^{2}}+w_{g},
\end{aligned}
$$




$$
\frac{\mathrm{d} \theta}{\mathrm{d} \xi}=\frac{\mathrm{d}^{2} \theta}{\mathrm{d} \xi^{2}}+\max (1, \phi)\left(q w_{v}-w_{g}\right),
$$

where

$$
w_{v}=-D_{v} y_{s} H\left(\theta-\theta_{v}\right), \quad w_{g}=-D_{a} \frac{y_{g} y_{o}}{1+\gamma\left(\theta-\theta_{f}\right)} \exp \left[\frac{\beta\left(\theta-\theta_{f}\right)}{1+\gamma\left(\theta-\theta_{f}\right)}\right],
$$

$D_{a}=t_{r} / t_{q}$ is the usual Damköhler number which we anticipate to be of order $\beta^{2}$, and $D_{v}=t_{r} / t_{v}$ is the Damköhler number associated to the vaporization. The objective of the following analysis is to obtain the flame speed, or equivalently the Damköhler number $D_{a}$, in the range of values of $t_{v}$ such that $D_{v}$ is of order unity. In the limit $D_{v} \gg 1$, i.e., $t_{r} / t_{v} \gg 1$, the solid fuel is converted rapidly to gas when the temperature reaches the value $T_{v}$, so we find the classical premixed flame. In the opposite case, $D_{v} \ll 1$, the vaporization proceeds very slowly resulting in a very small amount of gaseous fuel so the reactive wave cannot be self-sustained.

The boundary conditions for these equations are as follows:

$$
\begin{gathered}
\xi \rightarrow-\infty: \quad y_{s}=1, y_{o}=\phi^{-1}, y_{g}=\theta=0 ; \\
\xi \rightarrow+\infty: \quad \frac{\mathrm{d} y_{g}}{\mathrm{~d} \xi}=\frac{\mathrm{d} y_{o}}{\mathrm{~d} \xi}=\frac{\mathrm{d} \theta}{\mathrm{d} \xi}=0 .
\end{gathered}
$$

Moreover, we will impose that in first approximation $y_{g} y_{o}=0$ at $\xi \rightarrow+\infty$ due to the equilibrium condition.

The results obtained from the conservation laws for the regimes I and II in nondimensional form are as follows:

Rich mixture and full vaporization $\left(1<\phi<\phi_{1}\right)$

$$
y_{s, b}=y_{o, b}=0, \quad y_{g, b}=1-\phi^{-1}, \quad \theta_{b}=1-q \phi .
$$

\section{Lean mixture and full vaporization $\left(\phi_{0}<\phi<1\right)$}

$$
y_{s, b}=y_{g, b}=0, \quad y_{o, b}=\phi^{-1}-1, \quad \theta_{b}=1-q .
$$

\section{Preliminar description}

Before obtaining a detailed description of the flame structure it is convenient to give a general picture. If $\beta \gg 1$ the reaction zone is very thin compared with $l_{T}$. Since the problem is invariant under translations we can postulate that there exists a flame located at $\xi=0$, which appears as a discontinuity surface in the gradients of some variables. At the flame $\theta(0)=\theta_{f}, y_{s}(0)=y_{s, f}, y_{g}(0)=y_{g, f}$ and $y_{o}(0)=y_{o, f}$. Moreover the limit $\beta \rightarrow$ $+\infty$ implies that in first approximation $y_{g, f} y_{o, f}=0$. 
Figure 2 shows a general picture of the flow structure in the case of a lean mixture and full vaporization (regime II). We can identify three different regions and a reaction layer. In the region corresponding to $-\infty<\xi<\xi_{v}$, with $\xi_{v}$ defined by $\theta\left(\xi_{v}\right)=\theta_{v}$, the temperature is lower than the vaporization temperature $\theta_{v}$. Hence the mass fraction of solid fuel does not change in this region. However, $\theta \neq 0$ and $y_{g} \neq 0$ because of the heat and mass diffusion. Between $\xi=\xi_{v}$ and $\xi=0$ the vaporization results in decreasing values of $y_{s}$, while $y_{g}$ reaches a peak value due to the boundary condition $y_{g}(-\infty)=0$ and the gaseous fuel consumption in the reaction zone. The heat conduction from the reaction zone increases the temperature allowing the vaporization. Both pre-vaporization and vaporization sub-zones form the usual preheated zone found in the analysis of premixed flames and characterized by a convection-diffusion balance, and a frozen chemical reaction due to the low temperature and the large activation energy. The reaction sheet divides the flow into the preheat zone and the quasi-equilibrium zone, located downstream, where the remaining solid fuel is vaporized and the resulting gaseous fuel consumed if there is oxidant available. Due to the high temperature the consumption occurs immediately after the vaporization, so that $y_{g}(\xi) \approx 0$ for $\xi>0$.

In the regime I (rich mixture and full vaporization) this general picture is a bit different. The oxygen is completely consumed at the flame (Figure 3). Hence in the convective zone the flow is in equilibrium but the vaporization continues, resulting in a decreasing temperature profile.

\section{Analysis for unity Lewis numbers}

With the aim of reducing the mathematical complexity of the problem in favor of its physical aspects we will consider initially $L_{o}=L_{g}=1$. This assumption allows us to use two Shvab-Zel'dovich variables eliminating the Arrhenius term from all equations but one.

The equation for $y_{s}(2.20)$ can be integrated to obtain

$$
y_{s}= \begin{cases}-\infty<\xi \leq \xi_{v}: & 1, \\ \xi_{v} \leq \xi \leq+\infty: & \mathrm{e}^{D_{v}\left(\xi_{v}-\xi\right)}\end{cases}
$$

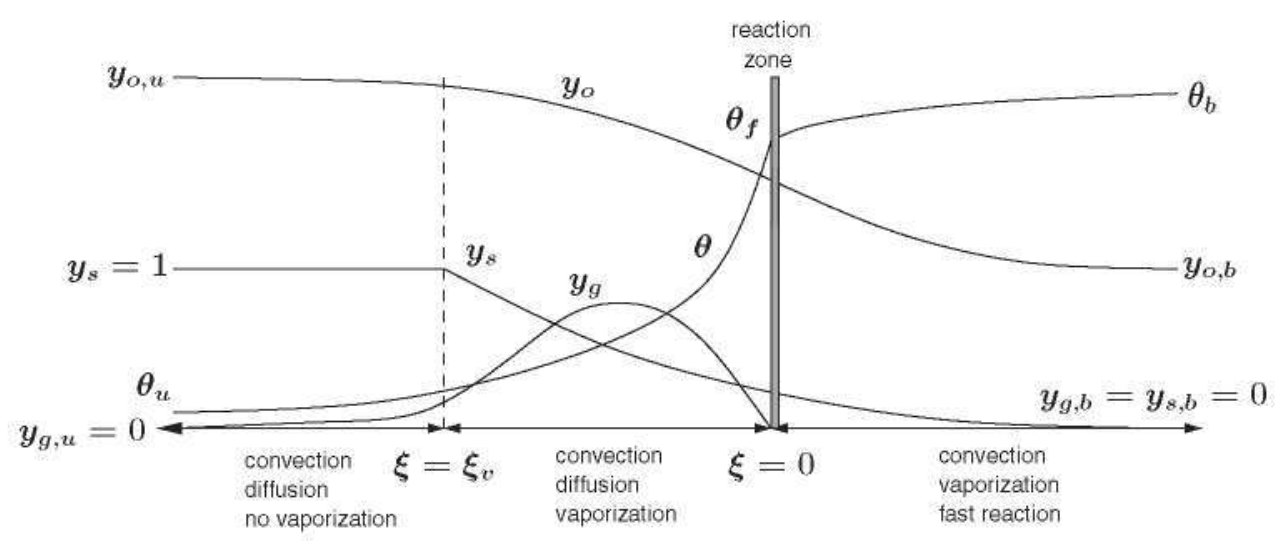

Figure 2. Schematic showing the general structure of the flow, at the scale of $I_{T}$, of a lean mixture. 


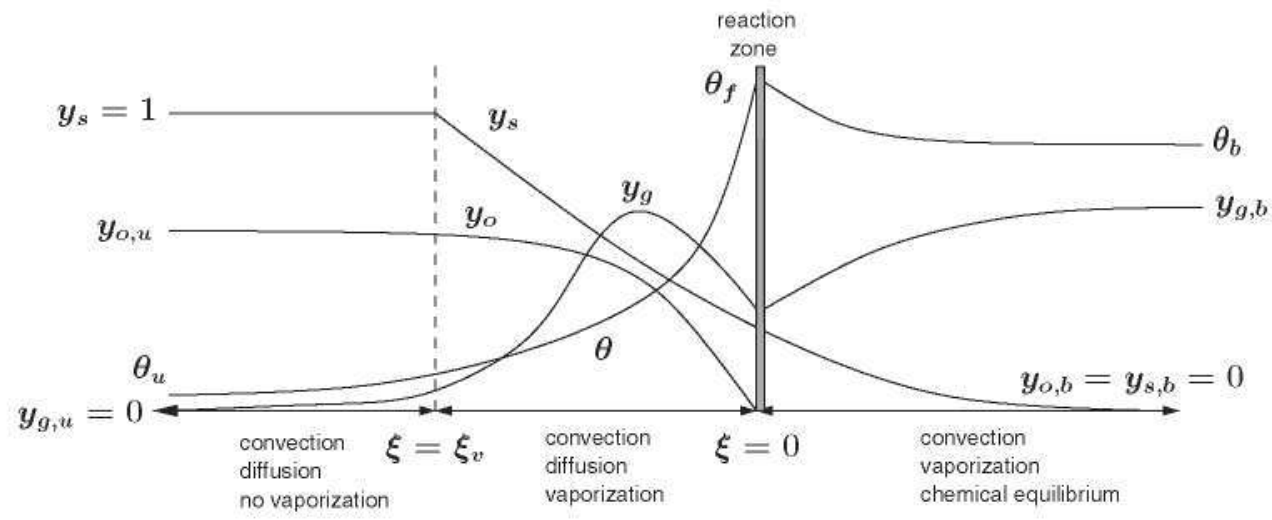

Figure 3. Schematic showing the general structure of the flow, at the scale of $I_{T}$, when the oxygen is fully depleted at the flame.

where $\xi_{v}$, still unknown, is the point upstream the flame where the temperature attains the value $\theta_{v}$.

If we define two Shvab-Zel'dovich variables $V$ and $U$ as

$$
V=q y_{g}+(1-q) y_{o}+\min \left(1, \phi^{-1}\right) \theta, \quad U=y_{g}-y_{o}
$$

we find from $(2.21-2.23)$ and $(2.25-2.28)$ that $V$ and $U$ verify the problems

$$
\begin{gathered}
\frac{\mathrm{d}^{2} V}{\mathrm{~d} \xi^{2}}-\frac{\mathrm{d} V}{\mathrm{~d} \xi}=0 ; \quad V(-\infty)=V(+\infty)=(1-q) \phi^{-1}, \\
\frac{\mathrm{d}^{2} U}{\mathrm{~d} \xi^{2}}-\frac{\mathrm{d} U}{\mathrm{~d} \xi}=-D_{v} y_{s} H\left(\theta-\theta_{v}\right) ; \quad U(-\infty)=-\phi^{-1}, U(+\infty)=1-\phi^{-1},
\end{gathered}
$$

whose solutions, after taking into account (3.1) and the continuity of $U$, are

$$
V=(1-q) \phi^{-1}, \quad U= \begin{cases}-\infty<\xi \leq \xi_{v}: & -\phi^{-1}+\frac{D_{v}}{1+D_{v}} \mathrm{e}^{\xi-\xi_{v}}, \\ \xi_{v} \leq \xi \leq+\infty: & 1-\phi^{-1}-\frac{1}{1+D_{v}} \mathrm{e}^{D_{v}\left(\xi_{v}-\xi\right)} .\end{cases}
$$

\section{Preheat zone $(\xi<0)$}

The analysis of the preheat zone can be done simultaneously for regimes I and II (rich and lean mixtures). In this zone the chemical reaction is frozen but not the vaporization. We can distinguish the pre-vaporization and vaporization sub-zones, where $\theta<\theta_{v}$ and $\theta>\theta_{v}$, respectively. The equation for the mass fraction of oxygen (2.22) is not affected by the vaporization and can be integrated to obtain:

$$
y_{o}=y_{o, f} \mathrm{e}^{\xi}+\phi^{-1}\left(1-\mathrm{e}^{\xi}\right),
$$

where $y_{o, f}$ stands for its value at the flame, which is related to the corresponding values of the mass fraction of gaseous fuel and temperature, $y_{g, f}$ and $\theta_{f}$, respectively, by $U(0)$ and $V(0)$ : 


$$
\begin{gathered}
y_{g, f}-y_{o, f}=1-\phi^{-1}-\frac{\mathrm{e}^{D_{v} \xi_{v}}}{1+D_{v}} . \\
\min \left(1, \phi^{-1}\right) \theta_{f}+y_{o, f}=\phi^{-1}-q\left(1-\frac{\mathrm{e}^{D_{v} \xi_{v}}}{1+D_{v}}\right) .
\end{gathered}
$$

Since, due to the limit $\beta \rightarrow+\infty$, in first approximation $y_{g f} y_{o f}=0$, (3.7) provides the value of the non-zero mass fraction.

The expressions for $y_{g}(\xi)$ and $\theta(\xi)$ in the preheat zone are obtained from (3.2), (3.5) and (3.6):

$$
\begin{gathered}
y_{g}= \begin{cases}-\infty<\xi \leq \xi_{v}: & y_{g, v} \mathrm{e}^{\xi-\xi_{v}}, \\
\xi_{v} \leq \xi \leq 0: & y_{g_{v}} \mathrm{e}^{\xi}+\left(1-\mathrm{e}^{\xi}\right)+\frac{\mathrm{e}^{D_{v} \xi v}}{1+D_{v}}\left(\mathrm{e}^{\xi}-\mathrm{e}^{-D_{v} \xi}\right),\end{cases} \\
\theta= \begin{cases}-\infty<\xi \leq \xi_{v}: & \theta_{v} \mathrm{e}^{\xi-\xi_{v}}, \\
\xi_{v} \leq \xi \leq 0: & \theta_{f} \mathrm{e}^{\xi}-q \max (1, \phi)\left(1-\mathrm{e}^{\xi}+\frac{\mathrm{e}^{D_{v} \xi_{v}}}{1+D_{v}}\left(\mathrm{e}^{\xi}-\mathrm{e}^{-D_{v} \xi}\right)\right),\end{cases}
\end{gathered}
$$

where $y_{g, v}$ and $\theta_{v}$ are related to $y_{g, f}$ and $\theta_{f}$, respectively, by

$$
\begin{gathered}
y_{g, v} \mathrm{e}^{-\xi_{v}}=y_{g_{v}}-1+\frac{D_{v} \mathrm{e}^{-\xi_{v}}+\mathrm{e}^{D_{v} \xi_{v}}}{1+D_{v}}, \\
\theta_{v} \mathrm{e}^{-\xi_{v}}=\theta_{f}+q \max (1, \phi)\left(1-\frac{D_{v} \mathrm{e}^{-\xi_{v}}+\mathrm{e}^{D_{v} \xi_{v}}}{1+D_{v}}\right) .
\end{gathered}
$$

This is all the information we can obtain from the analysis of the preheat zone that does not depend on which reactant is, in first approximation, fully depleted at the flame. The position of the vaporization starting point, $\xi_{v}$, and the flame speed will be obtained as result of the analysis of the downstream convective and reaction zones, respectively, which must be done taking into account whether $y_{g, f}=0$ or $y_{o_{f}}=0$.

\section{Lean mixtures $(\phi \leq 1)$}

If the mixture is lean $1-\phi^{-1}<0$ and then (see 3.7) $y_{g, f}-y_{o, f}<0$ which implies

$$
y_{g, f}=0, \quad y_{o f}=\frac{\mathrm{e}^{D_{v} \xi_{v}}}{1+D_{v}}+\phi^{-1}-1 .
$$

\section{Downstream convective zone}

In the downstream convective zone, $\xi>0$, the mass fraction of solid fuel is also given by (3.1) and, according to (3.13), there is still oxygen available. As the temperature is of the order of $\theta_{f}$ the vaporization continues producing gaseous fuel. Since the chemical reaction is not in equilibrium because $y_{o} y_{g} \neq 0$, neither frozen because $\theta \approx \theta_{f}$, we must retain both reaction terms in the equation for $y_{g}$ : 


$$
\frac{\mathrm{d} y_{g}}{\mathrm{~d} \xi}=\frac{\mathrm{d}^{2} y_{g}}{\mathrm{~d} \xi^{2}}+D_{v} y_{s}-D_{a} \frac{y_{g} y_{o}}{1+\gamma\left(\theta-\theta_{f}\right)} \exp \left[\frac{\beta\left(\theta-\theta_{f}\right)}{1+\gamma\left(\theta-\theta_{f}\right)}\right] .
$$

Close to the flame $y_{o} \sim y_{o_{1}}, y_{g} \sim y_{g, f} \approx 0$ and, due to the high temperature, the gaseous fuel produced by the vaporization is rapidly depleted by the combustion. Therefore, the gaseous fuel is not accumulated and $y_{g} \ll 1$ in this convective zone. We can assume that the gaseous fuel is in a steady state resulting from the balance between production and consumption. Then $y_{g}$ is given by

$$
y_{g}=\frac{D_{v}}{D_{a}} \frac{y_{s}}{y_{o}}\left(1+\gamma\left(\theta-\theta_{f}\right)\right) \exp \left[-\frac{\beta\left(\theta-\theta_{f}\right)}{1+\gamma\left(\theta-\theta_{f}\right)}\right] \text {, }
$$

with $y_{o}>y_{o, b} \neq 0$. As $D_{a} \sim \beta^{2}$ and $\theta>\theta_{f}, y_{g}$ is at most of order $\beta^{-2} \ll 1$.

With the steady-state approximation for $y_{g}$, the first approximations for $y_{o}$ and $\theta$ in the region $\xi>0$ are obtained from the Shvab-Zel'dovich variables with $y_{g}=0$ :

$$
y_{o}=\phi^{-1}-1+\frac{\mathrm{e}^{D_{v}\left(\xi_{v}-\xi\right)}}{1+D_{v}}, \quad \theta=(1-q)\left(1-\frac{\mathrm{e}^{D_{v}\left(\xi_{v}-\xi\right)}}{1+D_{v}}\right) .
$$

The last equation shows that $\theta$ is increasing, hence $\theta(\xi>0)>\theta_{f}>\theta_{v}$, and the vaporization and combustion continue in the convective region because although $y_{0}$ is decreasing its value is greater than $y_{o, b}=\phi^{-1}-1$.

From the continuity condition $\theta\left(0^{+}\right)=\theta\left(0^{-}\right)=\theta_{f}$ and (3.12) we obtain

$$
\theta_{v}+\frac{q D_{v}}{1+D_{v}}=\mathrm{e}^{\xi_{v}}-\frac{\mathrm{e}^{\left(1+D_{v}\right) \xi_{v}}}{1+D_{v}}
$$

that for given values of $\theta_{v}, q$ and $D_{v}$ allows us to compute $\xi_{v}$. For $D_{v} \gg 1$, i.e., very rapid vaporization compared with the flame propagation, $\xi_{v}=\ln \left(\theta_{v}+q\right)<0$. Moreover $D_{v}>D_{v, \min }=\theta_{v} /\left(1-\theta_{v}-q\right)$ for which $\xi_{v}=0$ (and $\left.\theta_{f}=\theta_{v}\right)$.

The values of $y_{o}$ and $\theta$ at the flame, given by

$$
y_{o, f}=\frac{\mathrm{e}^{D_{v} \xi_{v}}}{1+D_{v}}+\phi^{-1}-1, \quad \theta_{f}=(1-q)\left(1-\frac{\mathrm{e}^{D_{v} \xi_{v}}}{1+D_{v}}\right),
$$

are decreasing and increasing functions, respectively, of $D_{v}$. Therefore in the classical premixed flame limit, $D_{v} \rightarrow \infty$, all solid fuel has been converted into gas and burned at the flame so that $y_{o f f}$ and $\theta_{f}$ attain their minimum and maximum values, $\phi^{-1}-1$ and $1-q$, respectively.

\section{Analysis of the reaction zone}

The value of the Damköhler number, $D_{a}$, and, consequently, the flame speed is to be obtained from the analysis of the reaction zone. Since it is very thin, of order $\varepsilon=1 / \beta$, we will use a new space independent variable of order unity and the following expansions for $\theta, y_{g}, y_{o}$ and $y_{s}$ :

$$
\begin{gathered}
\eta=\xi / \varepsilon, \quad \theta=\theta_{f}+\varepsilon \varphi+o(\varepsilon), \quad y_{s}=y_{s, f}+\mathcal{O}(\varepsilon) \\
y_{g}=y_{g, f}+\varepsilon \min \left(1, \phi^{-1}\right) Y+o(\varepsilon), \quad y_{o}=y_{o f}+\varepsilon \min \left(1, \phi^{-1}\right) Z+o(\varepsilon) .
\end{gathered}
$$


with $y_{g_{t} f}=0$. When these expansions are taken to (3.2) to obtain the expansions of the Shvab-Zel'dovich variables, the following relations hold:

$$
q Y+(1-q) Z+\varphi=0, \quad Y-Z=\frac{D_{v} \mathrm{e}^{D_{v} \xi_{v}}}{1+D_{v}} \eta,
$$

and the Equation (2.21) for the mass fraction of gaseous fuel at leading order reads:

$$
\frac{\mathrm{d}^{2} Y}{\mathrm{~d} \eta^{2}}=\varepsilon^{2} D_{a}\left(y_{o, f}+\varepsilon Z\right) Y \mathrm{e}^{\varphi} .
$$

In this equation we have included two terms of the expansion of $y_{0}$ (Mitani, 1980; Williams, 1985, chap. 5), which allow us to extend the analysis for small values of $y_{o, f}$.

If we use the relations (3.20) to express $Z$ and $\varphi$ in terms of $Y$, the problem to be solved in the reaction zone is:

$$
\begin{gathered}
\frac{\mathrm{d}^{2} Y}{\mathrm{~d} \eta^{2}}=\frac{1}{2} \wedge Y\left(y_{o, f}-\varepsilon b \eta+\varepsilon Y\right) \mathrm{e}^{-Y+(1-q) b \eta}, \\
Y_{\eta}(-\infty)=-1+\mathrm{e}^{D_{v} \xi_{v}}, Y_{\eta}(+\infty)=0, \quad \lim _{\eta \rightarrow-\infty}\left[Y+\left(1-\mathrm{e}^{D_{v} \xi_{v}}\right) \eta\right]=0
\end{gathered}
$$

where $b=D_{v} \mathrm{e}^{D_{v} \xi_{v}} /\left(1+D_{v}\right)$ and $\Lambda=2 \varepsilon^{2} D_{a}$. The boundary conditions have been obtained from the matching conditions with the outer solutions. The additional matching condition determines $\Lambda$.

Once $\Lambda$ is obtained as a function of $D_{v}$ and the remaining parameters, the relation $D_{a}=$ $t_{r} / t_{q}=\left(D_{T, u} / U_{p}^{2}\right) B\left(T_{f}\right) \mathrm{e}^{-T_{a} / T_{f}}$ leads to:

$$
U_{p}=\left(2 \varepsilon^{2} \Lambda^{-1} D_{T, u} B\left(T_{f}\right) \mathrm{e}^{-T_{a} / T_{f}}\right)^{1 / 2} .
$$

Note that taking into account only the first term in the expansion of $y_{0}$ (see 3.21) we would obtain the problem formulated by Liñán (1974) in his analysis of the structure of counterflow diffusion flames in the premixed flame regime, for whose solution there are correlations available which would avoid us to perform the numerical integration of (3.22).

\section{Results and numerical example}

As an example we consider a lean mixture, $\phi=0.8$, of air and a volatile fuel with the same properties as methane (Poinsot and Veynante, 2005): $Q=5 \times 10^{7} \mathrm{~J} / \mathrm{Kg}, \quad v=4$, $c_{p}=1.45 \times 10^{3} \mathrm{~J} / \mathrm{K} \mathrm{Kg}, \quad T_{a}=1.1 \times 10^{4} \mathrm{~K}, \quad B_{1}=1.08 \times 10^{7} \mathrm{~s}^{-1}, \quad T_{u}=300 \mathrm{~K}, \quad T_{v}=450 \mathrm{~K}$ and $D_{T, u}=5 \times 10^{-5} \mathrm{~m}^{2} / \mathrm{s}$. These values lead to $Y_{s, u}=0.445 \times 10^{-1}, Y_{o, u}=0.223$, $y_{o, u}=1.25, T_{m}=1835 \mathrm{~K}$ and $\theta_{v}=0.977 \times 10^{-1}$. We will consider three values of the nondimensional vaporization heat $q=0,0.05$ and 0.1 .

The values of $\xi_{v}$ as a function of $D_{v}$, obtained by solving (3.17) for $D_{v} \in\left[D_{v, \text { min }}, 10\right]$, are plotted in Figure 4. For the same values of $D_{v}$ we compute $\theta_{f}$ and $y_{o f f}$ (Equation (3.18)), $y_{g, v}$ (Equation (3.11)) and $y_{s, f}$ (Equation (3.1)). These values are shown in Figure 5. For $D_{v} \geq 3$ all solid fuel has been vaporized ahead of the flame, $y_{s, f} \approx 0$, recovering the classical premixed flame. 


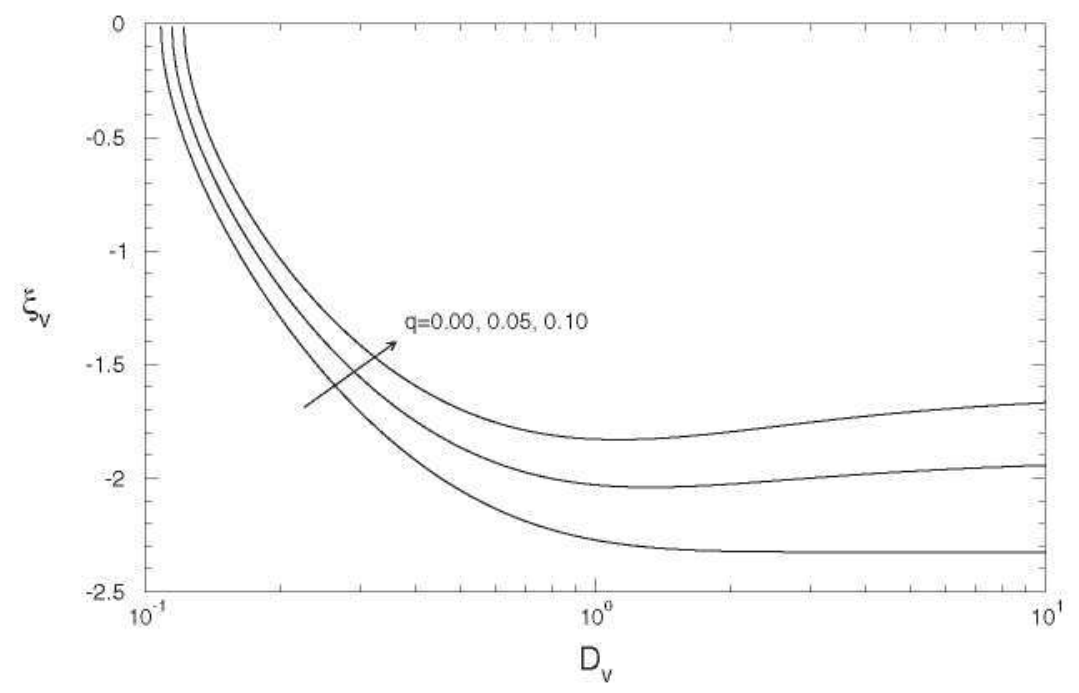

Figure 4. Lean mixtures. Vaporization starting point, $\xi_{v}$, as a function of $D_{v}$ (Equation 3.17).

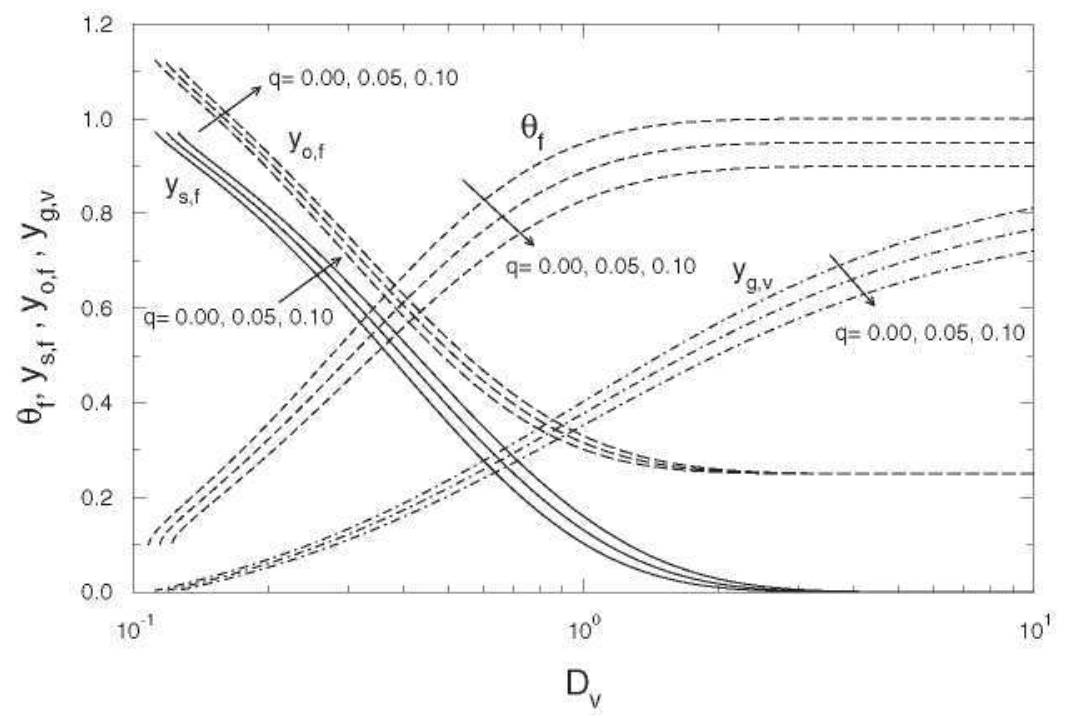

Figure 5. Lean mixtures. Flame temperature $\theta_{f}$ (Equation 3.18), and mass fractions $y_{s, f}$ and $y_{o, f}$ (Equations 3.1 and 3.13) and $y_{g, v}$ (Equation 3.11 with $y_{g, f}=0$ ) as functions of $D_{v}$.

Afterwards we compute $T_{f}=T_{u}+\theta_{f}\left(T_{m}-T_{u}\right), \quad \varepsilon=T_{f}^{2} / T_{a}\left(T_{m}-T_{u}\right), t_{q}^{-1}=B\left(T_{f}\right)$ $\mathrm{e}^{-T_{a} / T_{f}}, b=D_{v} \mathrm{e}^{D_{v} \xi_{v}} /\left(1+D_{v}\right)$ and solve the problem (3.22) to obtain the value of $\Lambda$. Once $\Lambda$ as a function of $D_{v}$ is known, since $\varepsilon$ and $t_{q}$ are also functions of $D_{v}$, the relations $\Lambda=2 \varepsilon^{2} D_{a}, D_{a}=t_{r} / t_{q}$ and $D_{v}=t_{r} / t_{v}$ lead to:

$$
t_{v}{ }^{-1}=2 \varepsilon^{2} D_{v} \Lambda^{-1} t_{q}^{-1}, \quad t_{r}^{-1}=t_{v}^{-1} / D_{v},
$$


which determines for a given value of $t_{v}$ the value of $D_{v}, D_{v}^{*}$, solution of the problem and the corresponding residence time. Once $D_{v}^{*}$ has been computed we obtain the flame speed as

$$
U_{p}=\left(2 \varepsilon^{2} \Lambda^{-1} D_{T, u} B\left(T_{f}\right) \mathrm{e}^{-T_{a} / T_{f}}\right)_{\left(D_{v}=D_{v}^{*}\right)}^{1 / 2}
$$

Figure 6 shows the residence and vaporization times as a function of $D_{v}$. For given values of $t_{v}$ and $q$ (solid line curves), which should be considered as data of the problem, we obtain the appropriate value of $D_{v}$ for which a steady solution exists and the corresponding dashed line curve determines $t_{r}$. From these values we obtain $U_{p}$ as a function of $t_{v}$, Figure 7. We see, as expected, that the maximum flame speed is attained for $q=0$ and $t_{v} \gtrless 3 \times 10^{-4}\left(D_{v} \gtrless 3\right)$, which corresponds to the adiabatic flame, i.e., without lack of energy due to the vaporization, and for $t_{v}$ sufficiently small to convert into gas all allowable solid fuel before reaching the flame, as shown in Figure 5. Note the strong reduction in the maximum value of the flame speed due to $q$ : $U_{p}(q=0.05) \approx 0.80 U_{p}(q=0)$ and $U_{p}(q=0.10) \approx 0.63 U_{p}(q=0)$.

Finally, the profiles of $\theta, y_{s}, y_{g}$ and $y_{o}$ for $q=0.10$ and $D_{v}=0.8$ are plotted in Figure 8. For these values $\xi_{v}=-1.80, \theta_{f}=0.78, y_{s, f}=0.23$ and $y_{o f f}=0.38$. The temperature evolves in the convective zone from its value at the flame to the final combustion value $\theta_{b}=0.9$, as result of the burning of the remaining solid fuel at the flame, $y_{s, f}$.

\section{Rich mixtures $(\phi>1)$}

Since the equivalence ratio of the mixture has been defined using the mass fraction of the solid fuel at the fresh end, when the mixture is rich the solid fuel is present in excess and

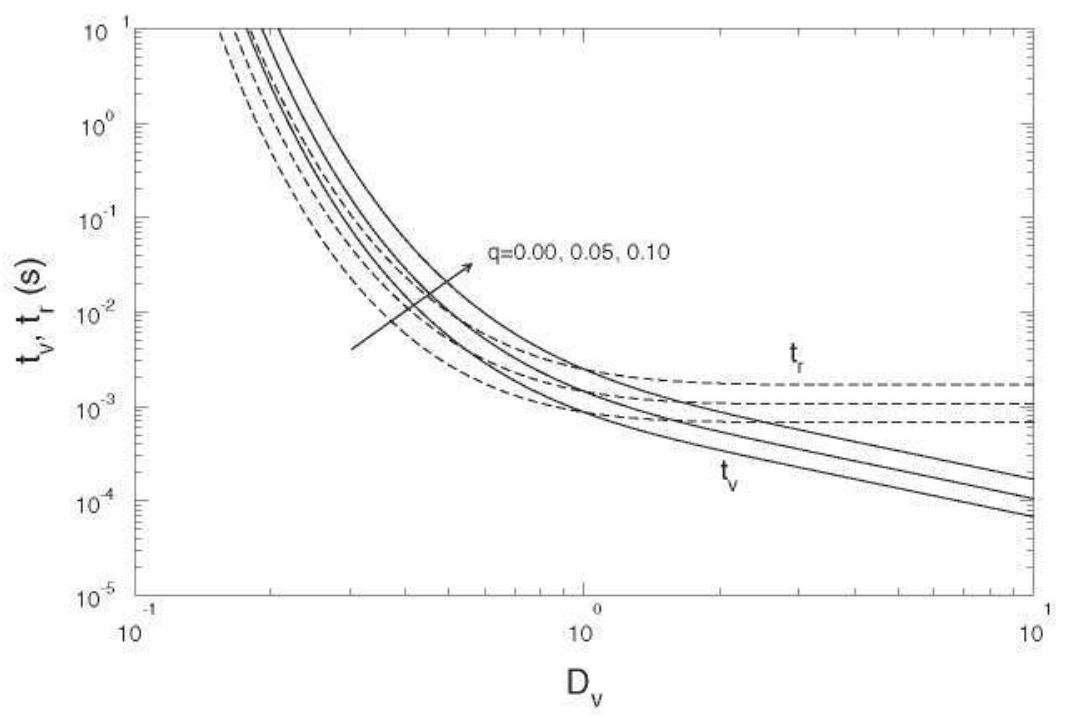

Figure 6. Lean mixtures. Vaporization and residence times, $t_{v}$ and $t_{r}$ as functions of $D_{v}$ (Equation 3.28 and $t_{r}=t_{v} D_{v}$ ). 


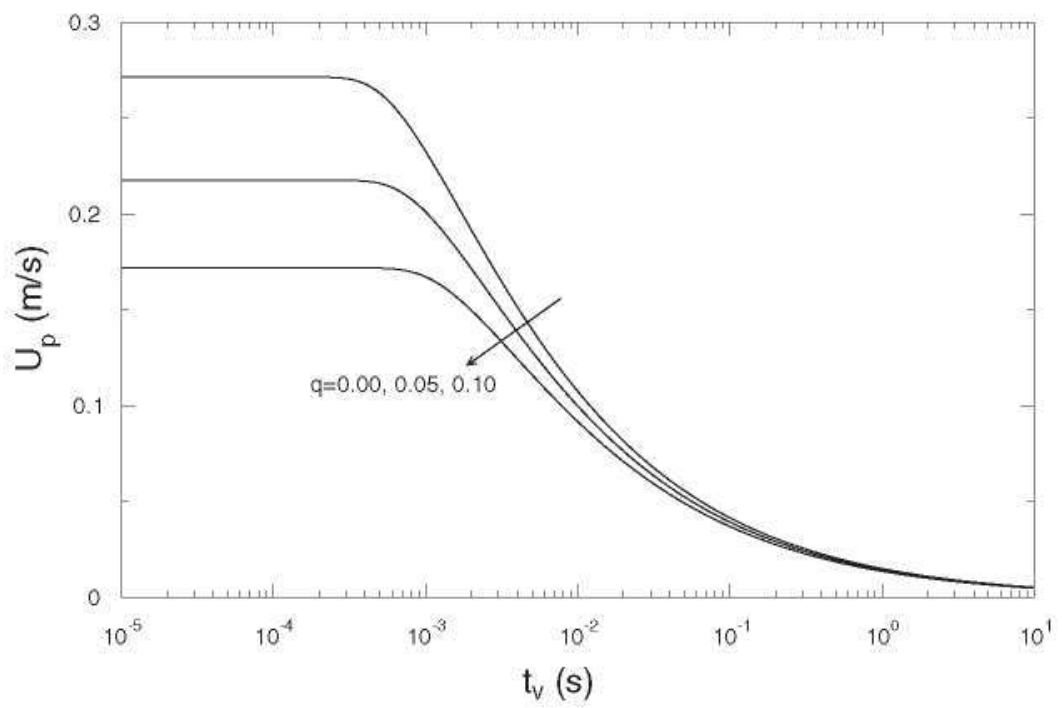

Figure 7. Lean mixtures. Flame speed as a function of the vaporization time $t_{\mathrm{v}}$.

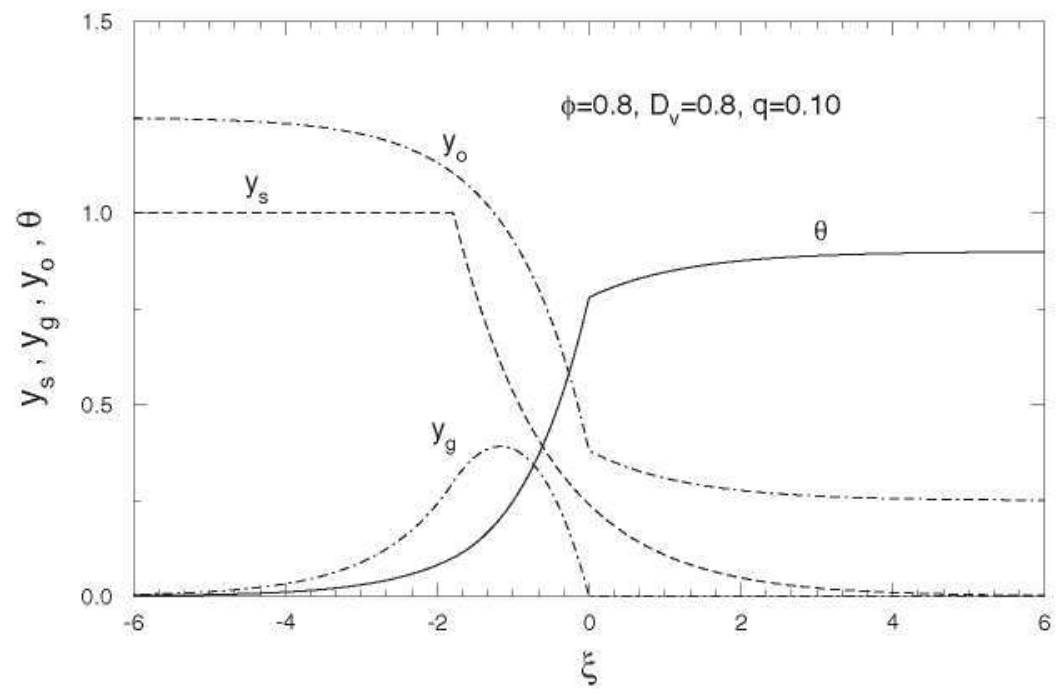

Figure 8. Lean mixtures. Profiles of mass fractions and temperature as functions of $\xi$ for $D_{v}=0.8$ and $q=0.10$.

the oxidant will be fully depleted. Hence at $\xi \rightarrow+\infty$ we will find $y_{o, b}=0$ and $y_{g, b} \neq 0$. However, as the chemical reaction takes place in the gaseous phase, the amount of solid fuel converted into gas upstream of the flame determines if the oxidant will be completely consumed at the flame or in the downstream convective zone. For sufficiently large values of $\phi$ the mass fraction of oxidant is small, and only a small amount of gaseous fuel is required for the full depletion of the oxidant at the flame. Hence, we can have $y_{o, f}=0$ for very rich mixtures even for small values of $D_{v}$. When the mixture is rich but not very far from the stoichiometry and $D_{v}$ is not sufficiently large, the amount of gaseous fuel 
produced before reaching the flame could be not enough to consume all the oxidant. In this case the gaseous fuel is, as in lean mixtures, the deficient reactant and we will find $y_{g, f}=0$ and $y_{o f}>0$. The remaining oxidant $y_{o f}$ is consumed in the downstream zone by the gaseous fuel produced behind the flame.

Given that in a rich mixture the deficient reactant can be the oxygen or the gaseous fuel, it is preferable to use instead of the classical division of mixtures as lean or rich, which takes into account the ratio of solid fuel to oxygen in the fresh mixture, the notion of gas-phase lean or rich mixtures, corresponding to which reactant is depleted at the flame: gaseous fuel $\left(y_{g f}=0\right)$ or oxygen $\left(y_{o, f}=0\right)$, respectively. The difference between these two types of mixtures arises not only by the incomplete vaporization of the solid fuel, but also by the different diffusive properties as it will be shown in $\S 4$. A lean mixture is always gas-phase lean (see (3.13)); however, if the mixture is rich $(\phi>1)$ the sign, positive or negative, of $U(0)$ determines the type of gas-phase mixture. The critical value of $D_{v}, D_{v, 0}$, dividing both regimes and for which the mixture is gas-phase stoichiometric $\left(y_{o f}=y_{g, f}=0\right)$ is given by

$$
1-\phi^{-1}=\frac{\mathrm{e}^{D_{r, 0} \xi_{r, 0}}}{1+D_{v, 0}}
$$

where $\xi_{v, 0}$ is the value of $\xi_{v}$ (Equation (3.33)) corresponding to $D_{v, 0}$. A mixture is gas phase rich if $D_{v}>D_{v, 0}$ or, equivalently, $1-\phi^{-1}>\mathrm{e}^{D_{v} \xi_{v}} /\left(1+D_{v}\right)$, while it is gas-phase lean in the opposite case.

\section{Gas-phase-rich mixtures $\left(\phi>1, D_{v}>D_{v, 0}, y_{o, f}=0\right)$}

When the mixture is gas-phase rich the mass fractions at the flame are $y_{o_{f} f}=0, y_{g_{f}}>0$ and $y_{s, f}>0$. Therefore the oxidant is completely consumed at the flame and there is not combustion reaction behind it. However, since $T_{b}>T_{v}$, the vaporization process continues in the convective zone. The distributions of $y_{s}(\xi), y_{o}(\xi), y_{g}(\xi)$ and $\theta(\xi)$ in the preheat zone are given by (3.1) and (3.6-3.10) with $y_{o f}=0$. The values of the temperature and gas fuel mass fraction at the flame, obtained from $U(0)$ and $V(0)$ with $y_{o, f}=0$, are given by

$$
y_{g_{f} f}=1-\phi^{-1}-\frac{\mathrm{e}^{D_{v} \xi_{v}}}{1+D_{v}}, \quad \theta_{f}=1-q \phi\left(1-\frac{\mathrm{e}^{D_{v} \xi_{v}}}{1+D_{v}}\right) \text {. }
$$

\section{Downstream convective zone}

As in first approximation the oxygen has been depleted at the flame, the distributions of $y_{g}$ and $\theta$ are given by (3.2) and (3.5) with $y_{o}=0$ :

$$
y_{g}=1-\phi^{-1}-\frac{\mathrm{e}^{D_{v}\left(\xi_{v}-\xi\right)}}{1+D_{v}}, \quad \theta=1-q \phi\left(1-\frac{\mathrm{e}^{D_{v}\left(\xi_{v}-\xi\right)}}{1+D_{v}}\right),
$$

while $y_{s}$ is given by (3.1). When the value of $\theta_{f}$ given by (3.31) is taken to (3.12) we obtain the equation to compute $\xi_{v}$ that can be written as

$$
\mathrm{e}^{\xi_{v}}=\left(\theta_{v}+q \phi \frac{D_{v}}{1+D_{v}}\right)
$$


Once $\xi_{v}$ is given as a function of $D_{v}>D_{v, 0}$, we can compute $\theta_{f}\left(D_{v}\right), y_{g, f}\left(D_{v}\right)$ and the remaining functions describing the outer solutions.

\section{Reaction zone}

The analysis of the reaction zone is done as in $\$ 3.2 .2$, with only minor changes. By using the space variable and expansions defined in (3.19) with $y_{o, f}=0$,

we obtain the equation for the leading term of the mass fraction of oxidant:

$$
\frac{\mathrm{d}^{2} Z}{\mathrm{~d} \eta^{2}}=\varepsilon^{2} D_{a}\left(y_{g, f}+\varepsilon \phi^{-1} Y\right) Z \mathrm{e}^{\varphi} .
$$

The order $\varepsilon$ expansion of the Shvab-Zel'dovich variables leads to

$$
q Y+(1-q) Z+\varphi=0, \quad Y-Z=\phi \frac{D_{v} \mathrm{e}^{D_{v} \xi_{v}}}{1+D_{v}} \eta
$$

from which we can express $Y$ and $\varphi$ in terms of $Z$. The boundary conditions result from the matching with the outer solutions. Finally the problem to be solved can be written as

$$
\begin{gathered}
\frac{\mathrm{d}^{2} Z}{\mathrm{~d} \eta^{2}}=\frac{1}{2} \Lambda Z\left(y_{g f}+\varepsilon b \eta+\varepsilon \phi^{-1} Z\right) \mathrm{e}^{-Z-q \phi b \eta}, \\
Z_{\eta}(-\infty)=-1, \quad Z_{\eta}(+\infty)=0, \quad \lim _{\eta \rightarrow-\infty}[Z+\eta]=0 .
\end{gathered}
$$

\section{Results and numerical example}

We consider the same values of the parameters as in $\$ 3.2 .3$ (lean mixtures), except the equivalence ratio $\phi=1.5$ for which $Y_{s, u}=0.804 \times 10^{-1}, Y_{o, u}=0.214, y_{o, u}=0.667, T_{m}=$ $2147 \mathrm{~K}$ and $\theta_{v}=0.812 \times 10^{-1}$. As before, we will consider three values of $q=0.00,0.05$ and 0.10 . The values of $D_{v, 0}$ are $0.325,0.348$ and 0.372 , respectively. Figures 9 and 10 show $\xi_{v}, \theta_{f}$ and the mass fractions $y_{s, f}, y_{g_{v} f}$ and $y_{g, v}$ as functions of $D_{v}$ in the range $\left.] D_{v, 0}, 10\right]$. The profiles of $\theta, y_{s}, y_{g}$ and $y_{o}$ for $q=0.10$ and $D_{v}=0.8$ are plotted in Figure 11. For these values we find that the mass fraction of solid fuel at the flame is $y_{s, f} \approx 0.26$, which is converted into gaseous fuel in the convective zone, but not burned due to the lack of oxygen, increasing the mass fraction $y_{g}$ and decreasing the temperature $\theta$ from their values at the flame.

The flame speed is obtained by solving the problem (3.38) to determine the function $\Lambda\left(D_{v}\right)$. As in Section $\S 3.2 .3$ for a given value of $t_{v}$ the relation (3.28) determines the appropriate value of $D_{v}, D_{v}^{*}$, and then the flame speed is given by (3.29). Figure 12 shows $t_{v}$ and $t_{r}=t_{v} D_{v}$ as a function of $D_{v}$ from which we obtain, for a given value of $t_{v}, D_{v}^{*}$, and $t_{r}\left(D_{v}^{*}\right)=D_{T, u} / U_{p}^{2}$. Figure 13 shows $U_{p}$ as a function of $t_{v}$. As expected $U_{p}$ is decreasing with $q$ and attains its maximum value for small values of $t_{v}$. In this case there is also a strong reduction in the flame speed due to $q: U_{p}(q=0.05) \approx 0.74 U_{p}(q=0)$ and $U_{p}(q=0.10) \approx 0.52 U_{p}(q=0)$. 


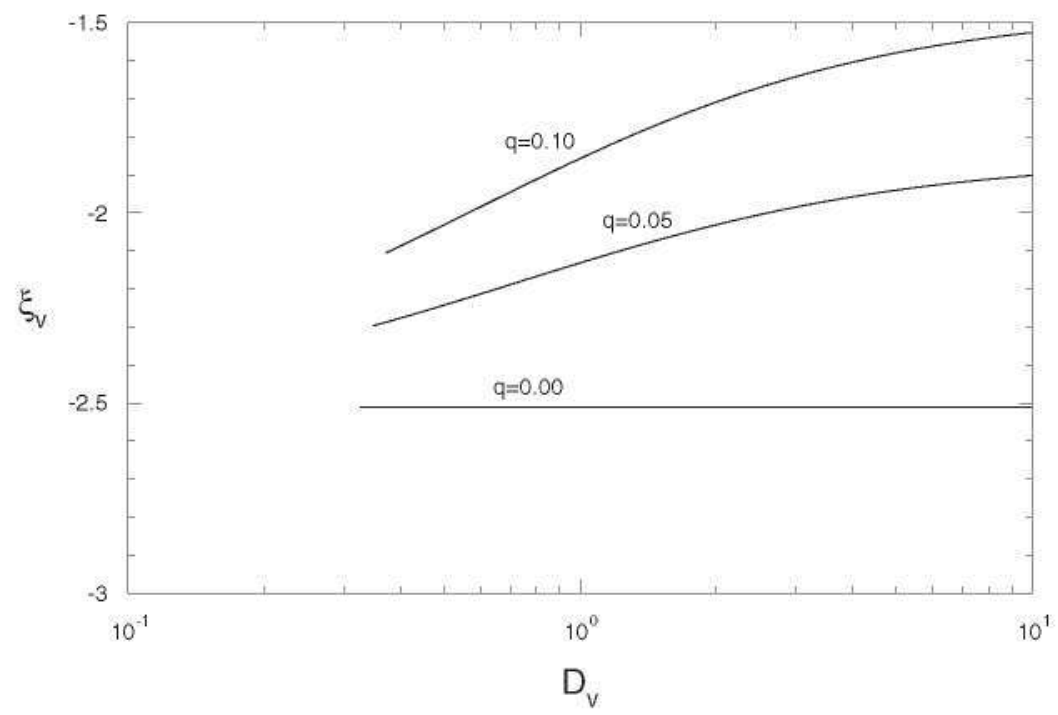

Figure 9. Gas-phase-rich mixtures. Vaporization starting point, $\xi_{v}$, as a function of $D_{v}$ (Equation 3.33).

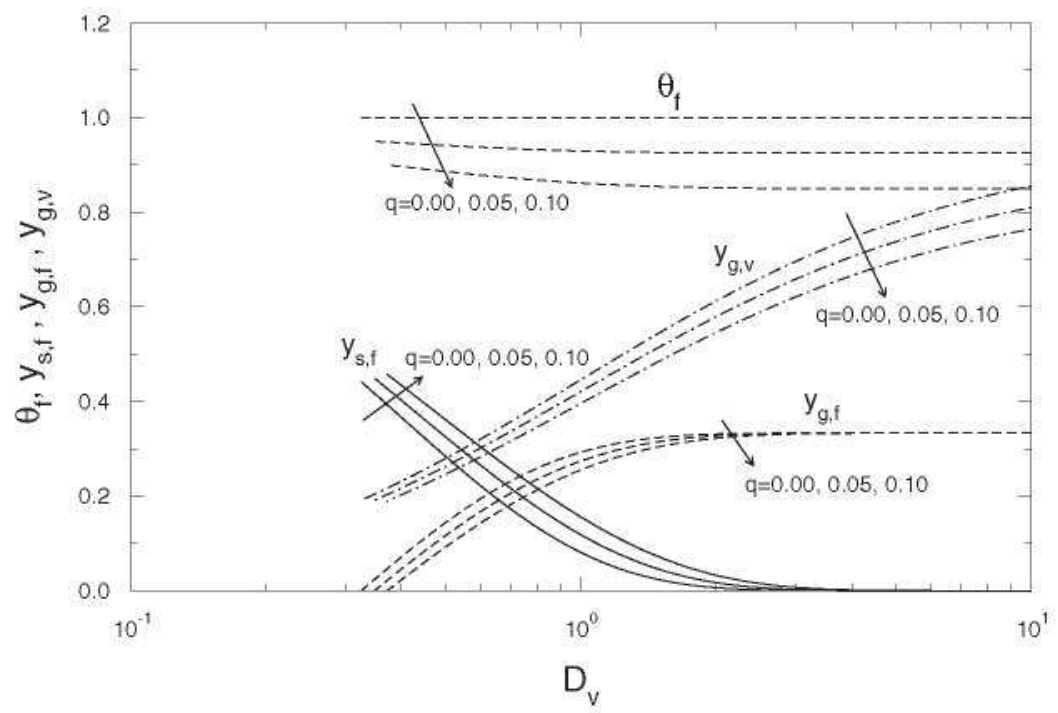

Figure 10. Gas-phase-rich mixtures. Flame temperature $\theta_{f}$ (Equation 3.31), and mass fractions $y_{s, f}$ and $y_{g, f}$ (Equations. 3.1 and 3.31) and $y_{g, v}$ (Equation 3.11) as functions of $D_{v}$.

\section{Gas-phase lean mixtures $\left(\phi>1, D_{v}<D_{v, 0}, y_{o, f}=0\right)$}

In this section, we will analyze the case of rich mixtures, but lean in the gas-phase, i.e., $\phi>1$, and $D_{v}<D_{y, 0}$, or equivalently $y_{g f}=0$. In the preheat zone the distributions of $y_{s}, y_{o}, y_{g}$ and $\theta$ are given by (3.1) and (3.6-3.10) with $y_{g, f}=0$. The values of $y_{o}$ and $\theta$ at the flame are 


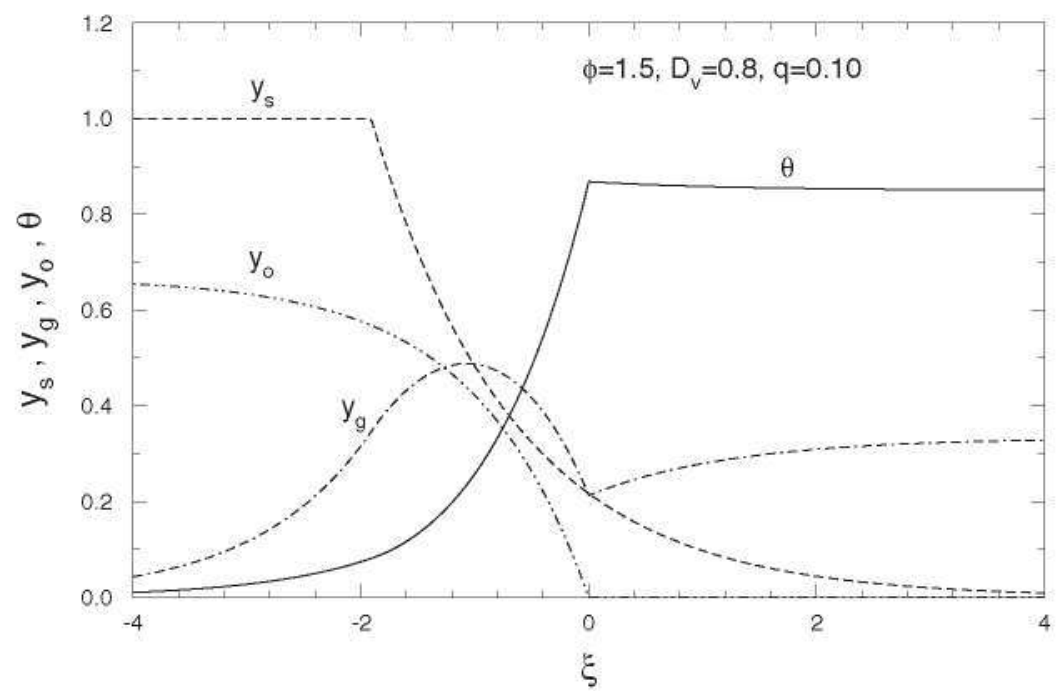

Figure 11. Gas-phase-rich mixtures. Mass fractions and temperature as functions of $\xi$ for $D_{v}=0.8$ and $q=0.10$.

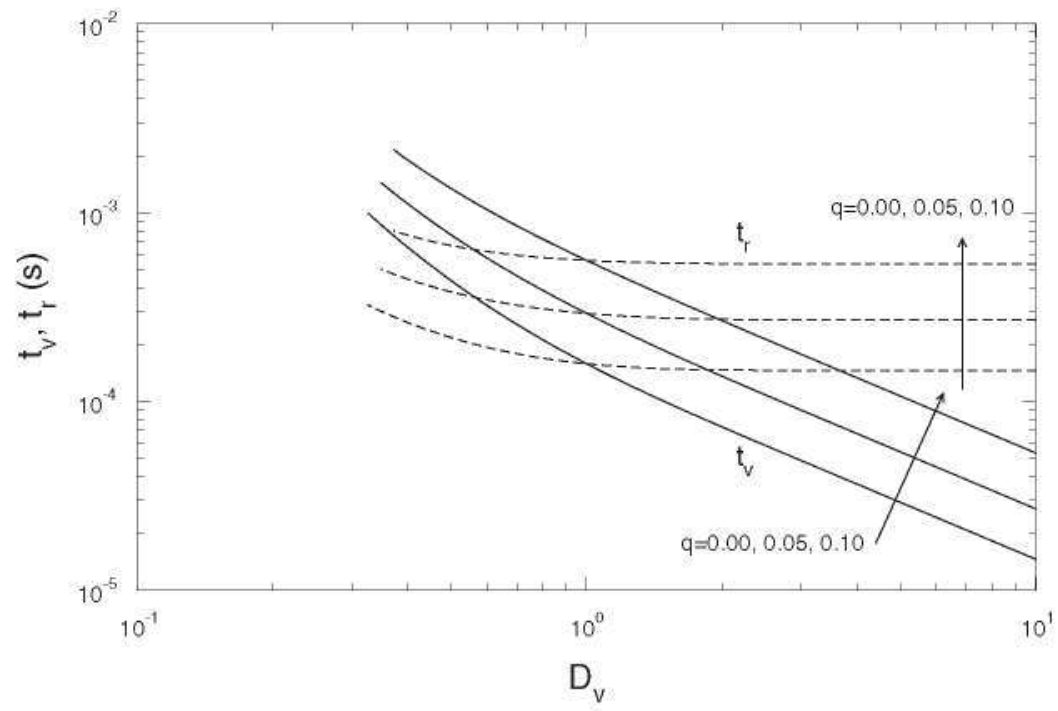

Figure 12. Gas-phase-rich mixtures. Vaporization and residence times, $t_{v}$ and $t_{r}$ as functions of $D_{v}$ (Equation 3.28 and $t_{r}=t_{v} D_{v}$ ).

$$
y_{o, f}=\frac{\mathrm{e}^{D_{v} \xi_{v}}}{1+D_{v}}-1+\phi^{-1}, \quad \theta_{f}=(1-q) \phi\left(1-\frac{\mathrm{e}^{D_{v} \xi_{v}}}{1+D_{v}}\right) .
$$

The equation to compute $\xi_{v}$ is again obtained from (3.12) and (3.39) leading to

$$
\phi^{-1} \theta_{v}+\frac{q D_{v}}{1+D_{v}}=\mathrm{e}^{\xi_{v}}-\frac{\mathrm{e}^{\left(1+D_{v}\right) \xi_{v}}}{1+D_{v}},
$$




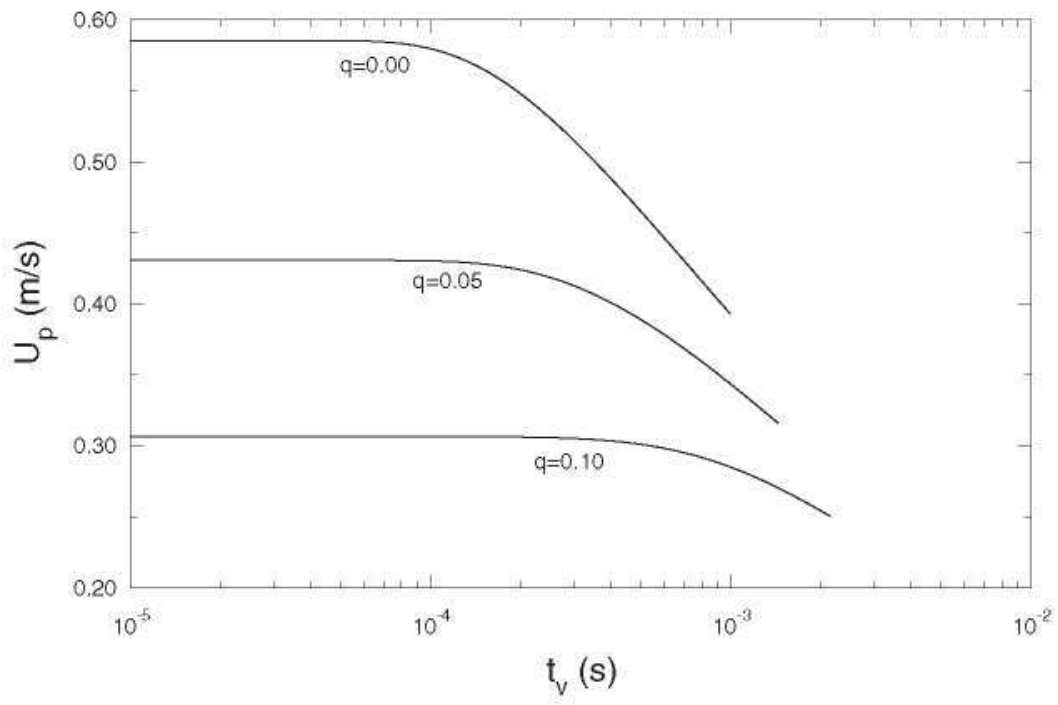

Figure 13. Gas-phase-rich mixtures. Flame speed as a function of the vaporization time $t_{v}$.

In the downstream convective zone there is oxidant available and, due to the high temperature, the reaction proceeds very rapidly. Hence, we will admit the steady-state hypothesis for $y_{g}$. Unlike what happened when $\phi<1$ and $y_{o, b}>0$, this process ends at $\xi=$ $\xi_{0}>0$ when all residual oxidant at the flame, $y_{o, f}$, has been fully consumed. As in first approximation $y_{g}\left(\xi_{0}\right)=y_{0}\left(\xi_{0}\right)=0$, the value of $\xi_{0}$ is given by $U\left(\xi_{0}\right)=0$, so that

$$
\xi_{0}=\xi_{v}-\frac{1}{D_{v}} \ln \left(\left(1-\phi^{-1}\right)\left(1+D_{v}\right)\right) .
$$

Therefore, downstream the flame we can distinguish a reactive sub-zone, corresponding to $0 \leq \xi \leq \xi_{0}$, followed by a properly convective zone, $\xi>\xi_{0}$, where we find chemical equilibrium due to the lack of oxygen although the vaporization continues producing gaseous fuel. In the reactive sub-zone $y_{g}$ (in steady state), $y_{0}$ and $\theta$ are given, in first approximation, by

$$
y_{g}=0, \quad y_{o}=\phi^{-1}-1+\frac{\mathrm{e}^{D_{v}\left(\xi_{v}-\xi\right)}}{1+D_{v}}, \quad \theta=(1-q) \phi\left(1-\frac{\mathrm{e}^{D_{v}\left(\xi_{v}-\xi\right)}}{1+D_{v}}\right),
$$

and the temperature is increasing due to the chemical reaction. In the convective zone, $\xi>\xi_{0}$, we find

$$
y_{g}=1-\phi^{-1}-\frac{\mathrm{e}^{D_{v}\left(\xi_{v}-\xi\right)}}{1+D_{v}}, \quad y_{o}=0, \quad \theta=1-q \phi\left(1-\frac{\mathrm{e}^{D_{v}\left(\xi_{v}-\xi\right)}}{1+D_{v}}\right),
$$

and the temperature decreases due to the vaporization of the solid fuel.

At $\xi=\xi_{0}$ there is a discontinuity on the gradients of the variables because when $\xi<\xi_{0}$ the mass fraction of oxygen $y_{o}$ is very small and then the steady-state hypothesis for $\tilde{y}_{g}$ is 
not valid. To remove it we should consider the full problem in a thin layer located around $\xi=\xi_{0}$. This layer has the same structure as the counterflow diffusion flame in the near equilibrium regime described by Liñán (1974), and does not affect to the flame propagation because the Damköhler number in the present case is much larger than the Damköhler number for extinction.

The analysis of the reaction zone is analogous to that of $\$ 3.2 .2$. From the space variable and expansions defined in (3.19) with $y_{g f}=0$, taking into account (3.37) and the matching with the outer solutions in the preheat zone, (3.9), and reactive sub-zone, $y_{g}=0$, we obtain the following problem for the mass fraction of gaseous fuel

$$
\begin{gathered}
\frac{\mathrm{d}^{2} Y}{\mathrm{~d} \eta^{2}}=\frac{1}{2} \Lambda Y\left(y_{o, f}-\varepsilon b \eta+\varepsilon \phi^{-1} Y\right) \mathrm{e}^{-Y+(1-q) \phi b \eta}, \\
Y_{\eta}(-\infty)=\phi\left(-1+\mathrm{e}^{D_{\nu} \xi_{v}}\right), \quad Y_{\eta}(+\infty)=0, \quad \lim _{\eta \rightarrow-\infty}\left[Y+\phi\left(1-\mathrm{e}^{D_{v} \xi_{v}}\right) \eta\right]=0 .
\end{gathered}
$$

The former analysis is only valid when $D_{v}$ is not very close to $D_{v, 0}$, so that $y_{o, f}$ and $\xi_{0}$ are both of order unity and the reactive sub-zone, where the gaseous fuel is in steady state and the oxygen is consumed by the chemical reaction, appears clearly defined. However, if $D_{v, 0}-D_{v} \sim \varepsilon, y_{o_{1} f}$ and $\xi_{0}$ are also of order $\varepsilon$. In this case the reactive sub-zone is embedded in the reaction zone, where all oxygen is consumed, and the model leading to the problem (3.44) has to be modified, because the steadystate hypothesis for $y_{g}$ does not apply.

The problem (3.44) must be replaced by a problem which describes the transition from the preheat to the convective zone. With the space variables and expansions given by (3.19), $y_{g, f}=0$ and taken into account (3.37) we obtain the following problem for $Z$ :

$$
\begin{gathered}
\frac{\mathrm{d}^{2} Z}{\mathrm{~d} \eta^{2}}=\frac{1}{2} \tilde{\Lambda}\left(Z+\frac{\phi y_{o f}}{\varepsilon}\right)(Z+\phi b \eta) \mathrm{e}^{-Z-q \phi b \eta} \\
Z_{\eta}(-\infty)=\phi\left(b / D_{v}-1\right), \quad Z_{\eta}(+\infty)=0, \quad \lim _{\eta \rightarrow-\infty}\left[Z-\phi\left(b / D_{v}-1\right) \eta\right]=0
\end{gathered}
$$

where $\tilde{\Lambda}=2 \phi^{-1} \varepsilon^{3} D_{a}$, and the boundary conditions have been obtained from the matching with the expansion of (3.6) for $\xi \rightarrow 0^{-}$and $y_{0}=0$ for $\xi \rightarrow 0^{+}$. This problem has to be solved for values of $D_{v}$ such that $D_{v, 0}-D_{v}=\mathcal{O}(\varepsilon)$ and, hence, $y_{o f f}=\mathcal{O}(\varepsilon)$, given by (3.39), while for smaller values of $D_{v}$ the problem (3.44) applies. Once $\tilde{\Lambda}$ is known the flame speed is given by

$$
U_{p}=\left(2 \varepsilon^{3} \phi^{-1} \tilde{\Lambda}^{-1} D_{T, u} B\left(T_{f}\right) \mathrm{e}^{-T_{a} / T_{f}}\right)^{1 / 2} .
$$

\section{Results and numerical example}

Figure 16 to 22 show the results for four different types of rich mixtures. Dotted lines corresponds to rich gas-phase mixtures, for which $D_{v}>D_{v, 0}$, whose results were given in $\S 3.4 .3$ for the full range of values of $D_{v}$. Thick dots indicate values for stoichiometric gasphase mixtures, $D_{v}=D_{v, 0}$. Results for quasi-stoichiometric lean gas-phase mixtures, 


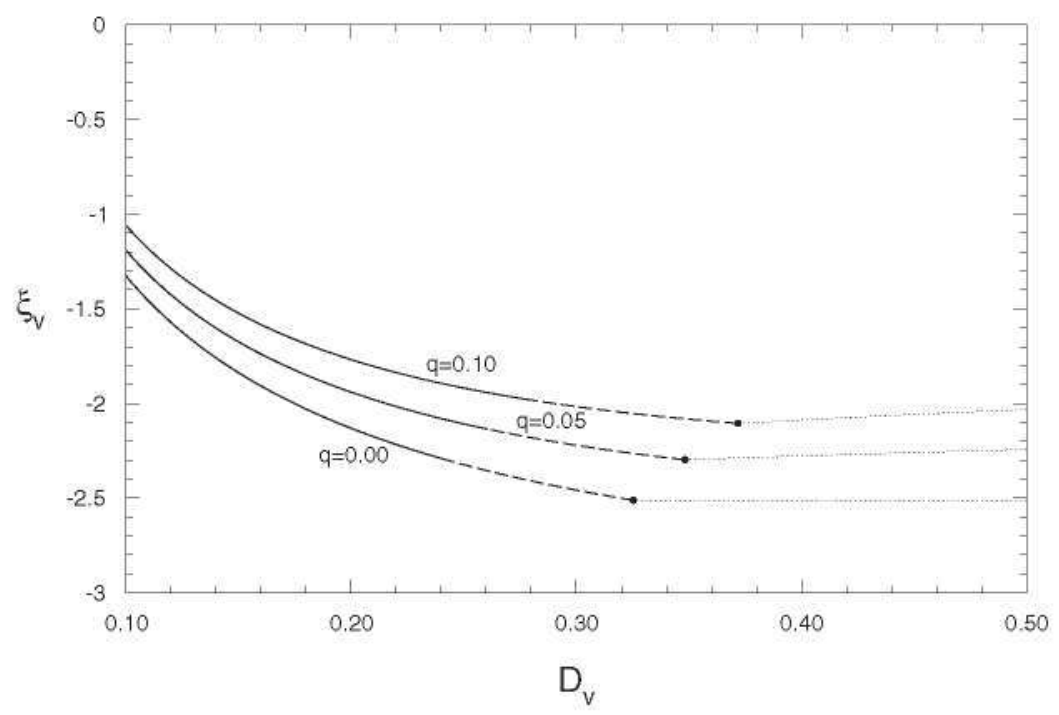

Figure 14. Gas-phase-lean mixtures. Vaporization starting point, $\xi_{v}$, as a function of $D_{v}$ (Equation 3.40).

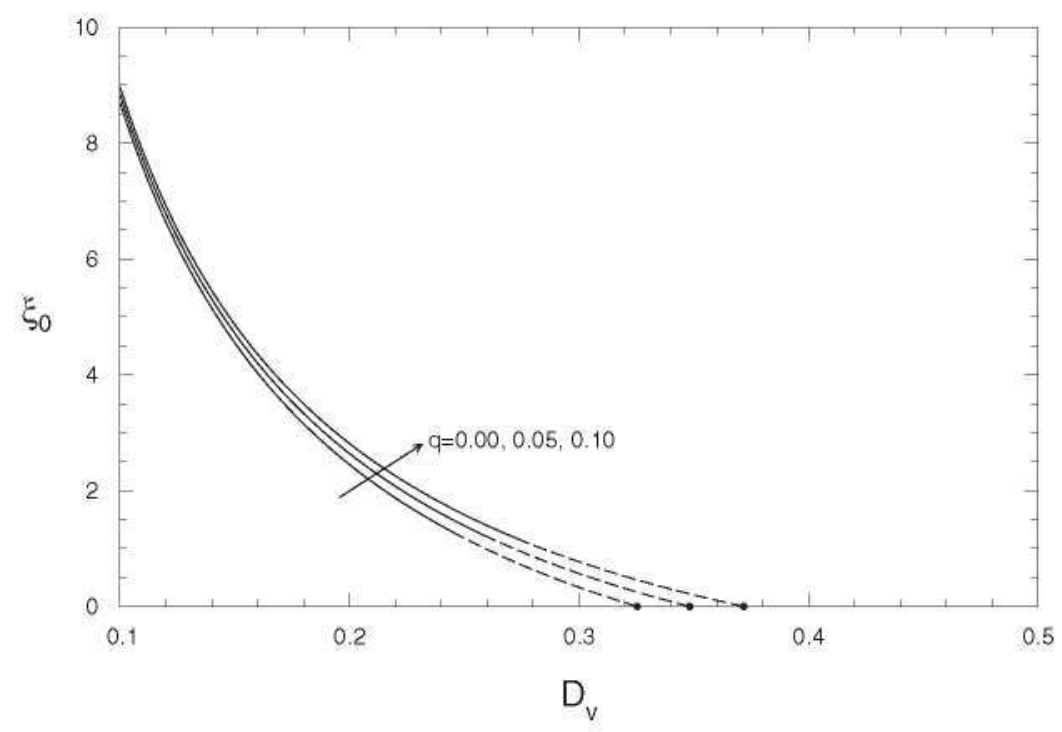

Figure 15. Gas-phase lean mixtures. Oxygen depletion point, $\xi_{0}$, as a function of $D_{v}$ (Equation 3.41).

$D_{v} \approx D_{v, 0}$, are showed with dashed lines. Finally, solid lines correspond to lean gas-phase mixtures, $D_{v}<D_{v, 0}$.

Figures 14-16 show $\xi_{v}, \xi_{0}, \theta_{f}$ and the mass fractions $y_{s, f}, y_{g_{f} f}$ and $y_{g, v}$ as functions of $D_{v}<D_{v, 0}$ and $q=0,0.05$ and 0.10 . The profiles of $\theta, y_{s}, y_{g}$ and $y_{o}$ for $q=0.10$ and $D_{v}=0.2$ are plotted in Figure 17. Figure 18 shows the residence and vaporization times, $t_{r}$ and $t_{v}$, as functions of $D_{v}$ computed from the solution of problems (3.44) and (3.45) in the appropriate range of values of 


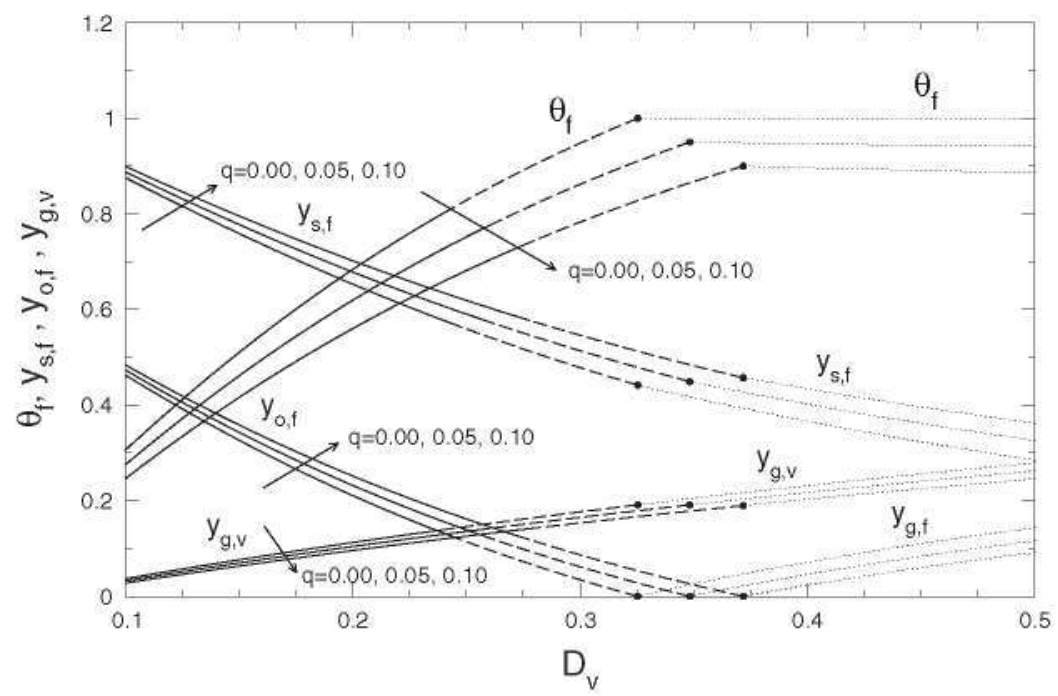

Figure 16. Gas-phase lean mixtures. Flame temperature $\theta_{f}$ (Equation 3.39), and mass fractions $y_{s, f}$ and $y_{o, f}$ (eqs. 3.1 and 3.39) and $y_{g, v}$ (Equation 3.11 with $y_{g, f}=0$ ) as functions of $D_{v}$.

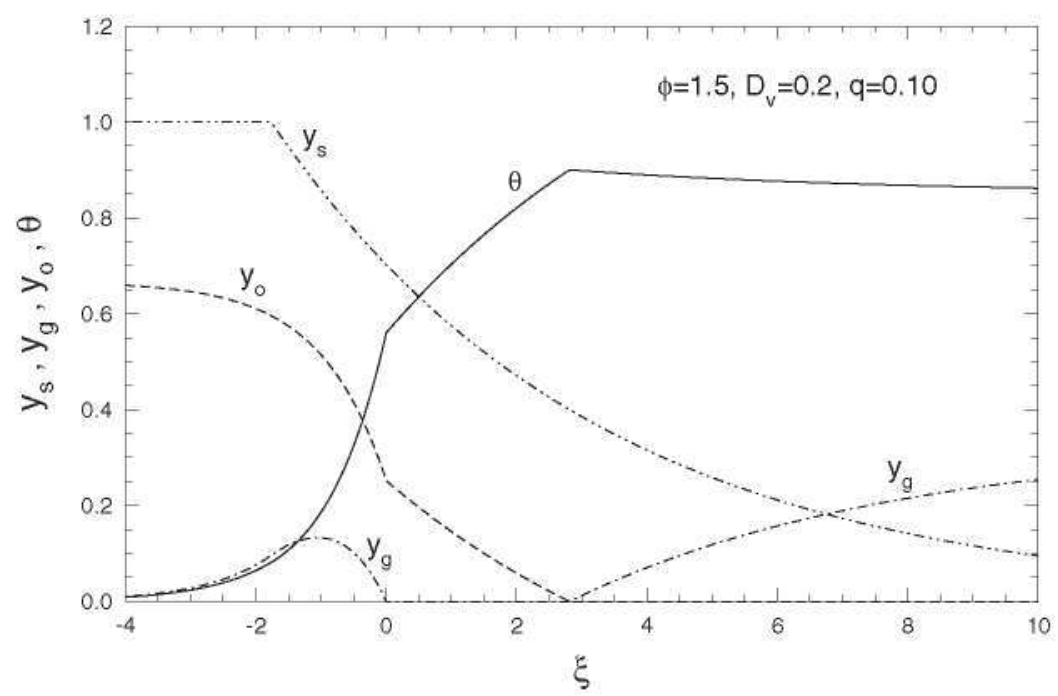

Figure 17. Gas-phase lean mixtures. Profiles of mass fractions and temperature as functions of $\xi$ for $\nabla=1.5, D_{v}=0.2$ and $q=0.10$.

$D_{v}<D_{v, 0}$. In Figure 19 we have plotted the values of the flame speed $U_{p}$ as a function of $t_{v}$ computed from Equations (3.29) and (3.46) .

\section{Analysis for non-unity Lewis numbers}

The analysis and results for non-unity Lewis numbers are openly available in figshare at http://doi.org/10.6084/m9.figshare.7825430. 


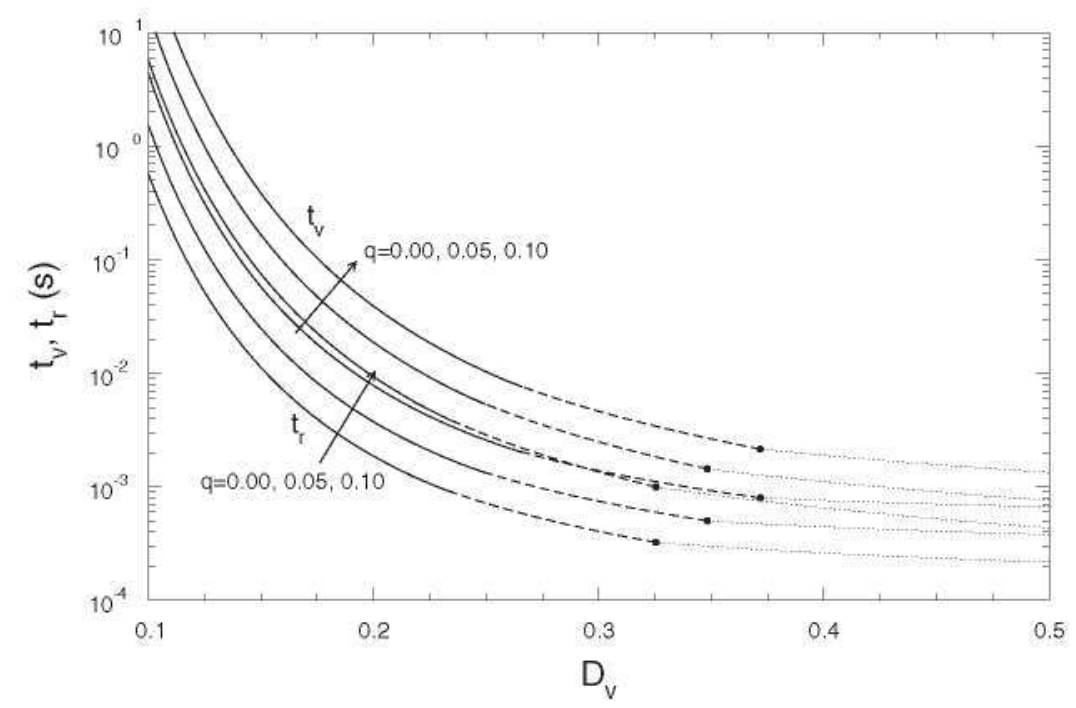

Figure 18. Gas-phase lean mixtures. Vaporization and residence times, $t_{v}$ and $t_{r}$ as functions of $D_{v}$.

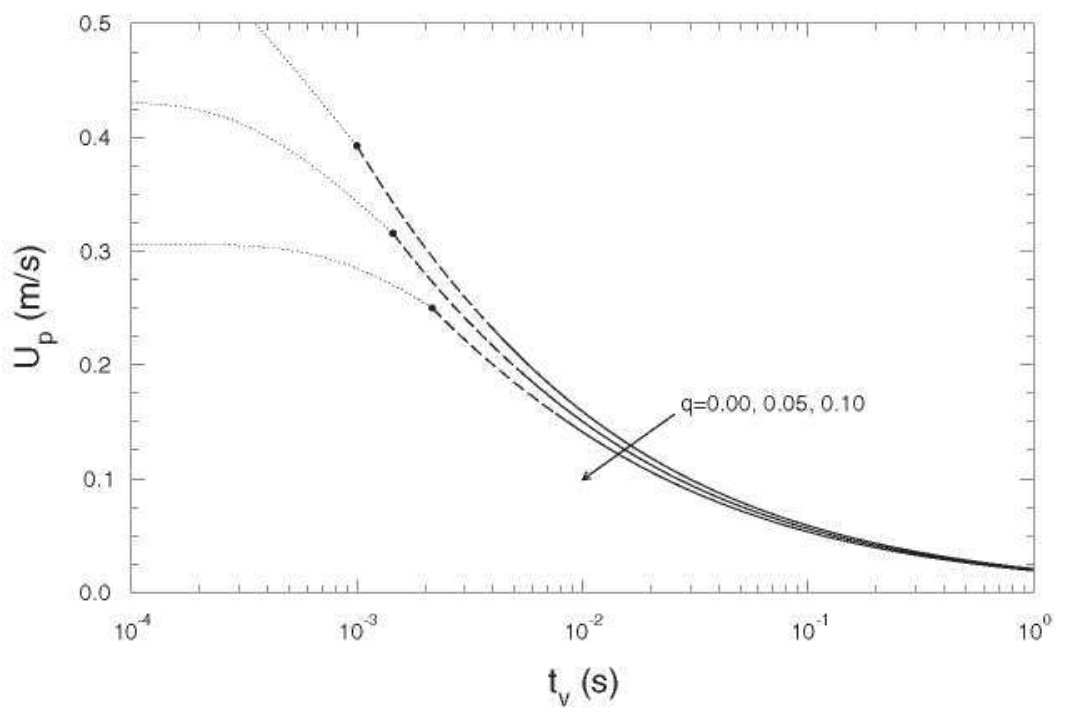

Figure 19. Gas-phase lean mixtures. Flame speed as a function of the vaporization time $t_{v}$.

\section{Summary and conclusions}

We have analyzed, using large activation energy asymptotics, the steady flame propagation through a cloud of solid fuel particles that, after vaporization, form a gaseous mixture of oxidant and fuel. The mass rate of the vaporization is modeled by a Heaviside function whose argument is the difference between the temperature and a threshold temperature, while we consider a global reaction of Arrhenius type with large activation energy to represent the combustion. We define two Damköhler numbers as the ratio of the residence time to the characteristic time of each reaction, namely $D_{v}$ and $D_{a}$ for vaporization 
and combustion, respectively. A classical thin reaction zone divides the flow field into two zones. Upstream the combustion is frozen but the vaporization produces gaseous fuel once the temperature rises to the threshold value. Downstream there is chemical equilibrium although, if the vaporization has not been completed before the reaction zone, there is a layer where the combustion of the remaining solid fuel occurs very rapidly due to the high temperature. Lean and rich mixtures, defined in terms of the initial solid fuel, have been considered although the analysis is restricted to those values of the equivalence ratio for which full vaporization is attained.

The structure of the flow and the flame speed have been obtained by using $D_{v}$ as main parameter, which plays a crucial role because it is directly related with the distribution of gaseous fuel in the flow. When the mixture is lean, the gaseous fuel is always the deficient reactant at the reaction zone. For moderately large values of $D_{v}$, i.e., small values of the characteristic time of vaporization, this is completed upstream the flame where all fuel is burned. For small values of $D_{v}$ the vaporization is completed in the convective zone downstream the flame. In this zone it is assumed that the gaseous fuel is burned at the same rate as it is produced by the vaporization due to the high temperature. The analysis shows that $D_{v}$ must be larger than a minimum value in order to reach the threshold temperature upstream the flame. Close to the end of vaporization, when the amount of solid fuel is very small and the distance between the particles is large, a diffusion flame could appear around each particle, hence the assumption of premixed combustion would not be valid. However, it is expected that this shortcoming does not have a great effect over the global results.

In the case of rich mixtures the deficient reactant can be oxygen or fuel depending on the value of $D_{v}$. There exists a critical value $D_{v, 0}$ for which the mixture is gas-phase stoichiometric. If $D_{v}>D_{v, 0}$ (fast vaporization) the deficient reactant is oxygen. Downstream the flame the vaporization continues, but there is not combustion due to the lack of oxygen. As a result the temperature decreases, being the final temperature lower than the flame temperature. When $D_{v}<D_{v, 0}$ the gaseous fuel is fully depleted at the flame, but vaporization and combustion persist downstream until all oxygen is consumed. In these cases there is an important increment on temperature behind the reaction zone followed by a slight decrease due to the heat absorbed by the vaporization. The fact that a rich mixture can be gas-phase lean or rich has been characterized (Seshadri et al., 1992) by an effective gas-phase equivalence ratio defined in terms of the flame temperature through an equivalent mass fraction of fuel. Since the flame temperature is an unknown of the problem we prefer to use the Damköhler number of vaporization to discriminate both situations, which, on the other hand, points directly to the cause of this situation.

The flame speed is computed in all cases by solving the appropriate reaction zone problem where $D_{a}$ plays the role of an eigenvalue. The results show that the flame speed decreases with both the characteristic time and the heat of vaporization by a nonnegligible amount.

Because of the advantages of using the Shvab-Zel'dovich coupling functions, we have thoroughly analyzed the case of both Lewis numbers equal to unity to describe the flow structure in the different regimes. The analysis when Lewis numbers are different from unity has been also carried out but presented in a concise way. It shows, as expected, that the flame speed increases with Lewis numbers. 
Radiation heat transfer has not been included in the formulation to keep it as simple as possible. However, Joulin and other researchers (e.g., Deshaies and Joulin, 1986; Joulin and Deshaies, 1986; Joulin, 1987; Liberman et al., 2015; Proust et al., 2017) have shown that thermal radiation can increase the flame speed, playing a significant role in dust explosions, and, hence, it will be addressed in a forthcoming paper in which a more detailed model is developed.

\section{References}

Bermúdez, A., Ferrín, J.L., and Liñán, A. 2007. The modelling of the generation of volatiles, H2 and $\mathrm{CO}$, and their simultaneous diffusion controlled oxidation, in pulverized coal furnaces. Combust. Theor. Model., 11(6), 949-976. doi: 10.1080/13647830701316640.

Bermúdez, A., Ferrín, J.L., Liñán, A., and Saavedra, L. 2011. Numerical simulation of group combustion of pulverized coal. Combust. Flame, 158(9), 1852-1865. doi: 10.1016/j.combustflame.2011.02.002.

Bidabadi, M., Beidaghy Dizaji, H., Faraji Dizaji, F., and Mostafavi, S.A. 2015. A parametric study of lycopodium dust flame. J. Eng. Math., 92(1), 147-165. doi: 10.1007/s10665-014-9769-3.

Bidabadi, M., Haghiri, A., and Rahbari, A. 2010. The effect of Lewis and Damköhler numbers on the flame propagation through micro-organic dust particles. Int. J. Therm. Sci., 49(3), 534-542. doi: 10.1016/j.ijthermalsci.2009.10.002.

Cloney, C.T., Ripley, R.C., Pegg, M.J., and Amyotte, P.R. 2018. Laminar burning velocity and structure of coal dust flames using a unity Lewis number CFD model. Combust. Flame, 190, 87-102. doi: 10.1016/j.combustflame.2017.11.010.

Deshaies, B., and Joulin, G. 1986. Radiative transfer as a propagation mechanism for rich flames of reactive suspensions. Siam Journal on Applied Mathematics, 46(4), 561-581.

Eckhoff, R.K. 1997. Dust Explosions in the Process Industries, Butterworth-Heinemann, Oxford, $2^{\text {nd }}$ ed.

Gao, W., Mogi, T., Yu, J., Yan, X., Sun, J., and Dobashi, R. 2015. Flame propagation mechanisms in dust explosions. J. Loss Prevent. Proc. Ind., 36, 186-194. doi: 10.1016/j.jlp.2014.12.021.

Joulin, G. 1987. Temperature-Lags and Radiative Transfer in Particle-Laden Gaseous Flames Part I: Steady Planar Fronts. Combustion Science and Technology, 52(4-6), 377-395.

Joulin, G., and Deshaies, B. 1986. On radiation-affected flame propagation in gaseous mixtures seeded with inert particles. Combustion Science and Technology, 47(5-6), 299-315.

Krazinski, J.L., Buckius, R.O., and Krier, H. 1979. Coal dust flames: A review and development of a model for flame propagation. Prog. Energy Combust. Sci., 5(1), 31-71. doi: 10.1016/03601285(79)90018-2.

Liberman, M., Ivanov, M., and Kiverin, A. 2015. Radiation heat transfer in particle-laden gaseous flame: flame acceleration and triggering detonation. Acta Astronautica, 115, 82-93.

Liñán, A. 1974. The asymptotic structure of counterflow diffusion flames for large activation energies. Acta Astronaut., 1(7-8), 1007-1039. doi: 10.1016/0094-5765(74)90066-6.

Mitani, T. 1980. Propagation velocities of two-reactant flames. Combust. Sci. Technol., 21(3-4), 175-177. doi: $10.1080 / 00102208008946931$.

Nakamura, M., Akamatsu, F., Kurose, R., and Katsuki, M. 2005. Combustion mechanism of liquid fuel spray in a gaseous flame. Phys. Fluids, 17(12), 1-14. doi: 10.1063/1.2140294.

Poinsot, T., and Veynante, D. 2005. Theoretical and Numerical Combustion, Toulouse, $3^{\text {rd }}$ ed.

Proust, C. 2006a. A few fundamental aspects about ignition and flame propagation in dust clouds. J. Loss Prevent. Proc. Ind., 19(2-3), 104-120. doi: 10.1016/j.jlp.2005.06.035.

Proust, C. 2006b. Flame propagation and combustion in some dust-air mixtures. J. Loss Prevent. Proc. Ind., 19(1), 89-100. doi: 10.1016/j.jlp.2005.06.026.

Proust, C., Ben Moussa, R., Guessasma, M., Saleh, K., and Fortin, J. 2017. Thermal radiation in dust flame propagation. Journal of Loss Prevention in the Process Industries, 49, 896-904.

Rockwell, S.R., and Rangwala, A.S. 2013. Modeling of dust air flames. Fire Saf. J., 59, 22-29. doi: 10.1016/j.firesaf.2013.03.006. 
Seshadri, K., Berlad, A.L., and Tangirala, V. 1992. The structure of premixed particle- cloud flames. Combust. Flame, 89(3-4), 333-342. doi: 10.1016/0010-2180(92)90019-L.

Slezak, S.E., Buckius, R.O., and Krier, H. 1985. A model of flame propagation in rich mixtures of coal dust in air. Combust. Flame, 59(3), 251-265. doi: 10.1016/0010-2180(85)90129-4.

Smoot, L.D., and Horton, M.D. 1977. Propagation of laminar pulverized coal-air flames. Prog. Energy Combust. Sci., 3(4), 235-258. doi: 10.1016/0360-1285(77)90014-4.

Williams, F.A. 1985. Combustion Theory, Benjamin Cummings, Menlo Park CA, $2^{\text {nd }}$ ed. 